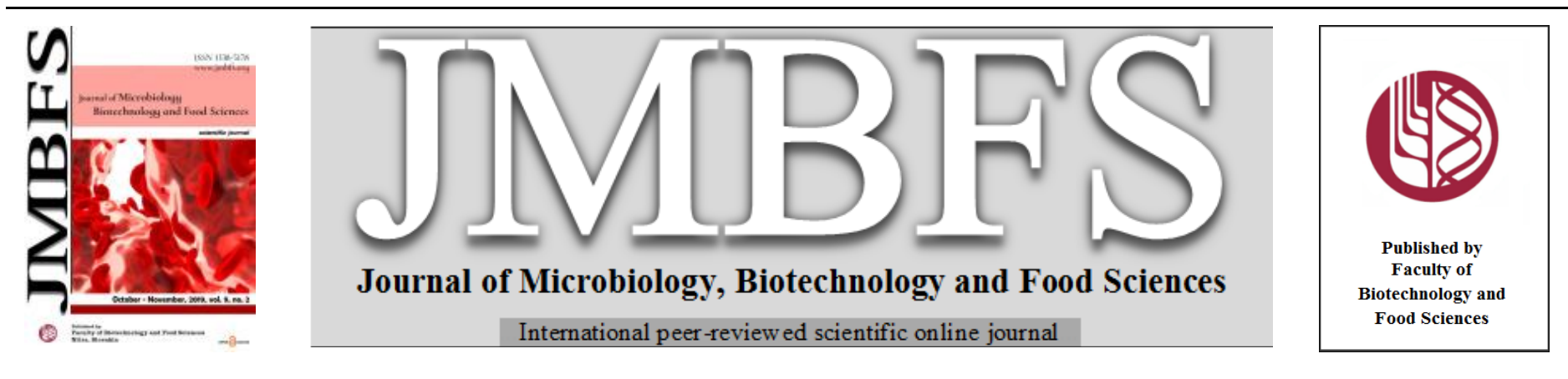

\title{
HYDROTHERMAL TREATMENTS FOR PADDY TO IMPROVE PHYSICOCHEMICAL QUALITY OF BROWN RICE
}

\author{
Kaur A ${ }^{1}$, Suresh Bhise ${ }^{2 *}$, Mandeep Kaur ${ }^{1}$ \\ Address(es): \\ ${ }^{1}$ Department of Food Science and Technology, Punjab Agricultural University, Ludhiana. \\ ${ }^{2}$ College of Food Processing Technology \& Bio-Energy, Anand Agricultural University, Anand, Gujarat.
}

*Corresponding author: sureshbhise cft@ yahoo.co.in

doi: 10.15414/jmbfs.2020.9.5.913-926

\section{ARTICLE INFO}

Received 22. 5. 2019

Revised 16. 9. 2019

Accepted 22. 10. 2019

Published 1. 4. 2020

Regular article

OPEN OACCESS

\begin{abstract}
The rice varieties namely PR-115, PR-118 and Punjab Mehak were subjected to three different treatments to improve physicochemical qualities of brown rice. Paddy milled to brown rice and then stored at room temperature in four different types of packaging materials. Brown rice was assessed periodically for changes in physicochemical characteristics. Milling quality improved with treatments. Moisture content, thousand kernel weight, bulk density and L/B ratio differ significantly for treatments and storage period. Protein, ash and fat content remain constant throughout the storage period. Color, appearance and amylose content improved with treatments yielding better quality brown rice. Storage resulted in lowering the levels of free fatty acids and peroxide value with treatments. Hot water treatment followed by steaming for $15 \mathrm{~min}$ was found to be best among all other treatments. Punjab Mehak gave best response to treatments and hence retained better functional properties upon storage. Packaging in plastic bag under vacuum was found to be the best packaging material for treated samples as compared to control. Overall hydrothermal treatments proved to be functional in improving physicochemical and functional quality of brown rice.
\end{abstract}

Keywords: Treatment, soaking, steaming, packaging, storage, brown rice, shelf life

\section{INTRODUCTION}

Rice accounts for over 20 per cent of global calorie intake. Over 90 per cent of the world's rice was produced and consumed in the Asian Region by 6 countries (China, India, Indonesia, Bangladesh, Vietnam and Japan) comprising 80 per cent of the world's production and consumption. Comparison of various characteristics before and after parboiling was made by Vitti et al. (1975) and reported increase in thiamine content with parboiling. Parboiling of rice reduced the development of free fatty acid in rice bran (Shaheen et al.1975) and bran of parboiled rice could be stored for more than 1 month without serious deterioration of oil and bran quality. Steam treatment caused partial denaturation of soluble protein fraction, especially those soluble in water and salt (Tarosova et al. 1971). Ash content increased slightly with time of steeping. Protein content was not much affected. The vitamin B content was increased three folds due to hydrothermal treatment.

It was found that riboflavin, magnesium and manganese contents were not affected by soaking and steaming treatments. Thiamine, calcium and phosphorous levels increased and this increase appeared to be caused by an inward diffusion of these substances during steaming and soaking. Steaming for 1 minute at $118^{\circ} \mathrm{C}$ and $1.94 \mathrm{~kg} / \mathrm{cm}^{2}$ gave satisfactory free fatty acids levels and peroxide concentration without effecting cooking quality was reported by Kibuuka (1979). It reduced the carbonyl contents (by 42.74 per cent) compared with original brown rice. Otegbayo et al. (2001) reported that parboiling reduced the breakage, fat, protein and amylose content of the rice whereas during cooking, water uptake and thiamine contents were increased. Parboiling could be a way of improving vitamin content and milling properties of rice.

Brown rice was an unmilled rice or partly milled rice. It's a kind of whole grain. During polishing 15 per cent of protein, 85 per cent of the fat, 90 per cent of the calcium, 75 per cent of the phosphorus, 80 per cent of thiamine, 70 per cent of riboflavin, 90 per cent of pyridoxine, 60 per cent of iron, 68 per cent of niacin was removed. Now a day's nutritionist and dieticians are recommending the brown rice for all round nutrition. It reduces asthma due to antioxidants and phytonutrients, helps prevent cancer (Tian et al, 2004), helps increase energy levels (fiber), helps in healthy bone formation, prevent atherosclerosis and gallstones. The brown rice had limited shelf life; keeping this in view current study was undertaken.

The physico-chemical properties of milled rice obtained from parboiling rough rice and brown rice need to be clearly documented; hence, this study was undertaken to standardize the parboiling treatments to extend the shelf life of brown rice and study the effect of hydrothermal treatments on physicochemical quality of brown rice.

\section{MATERIAL AND METHODS}

The present study was carried out in Department of Food Science and Technology, College of Agriculture, Punjab Agricultural University, Ludhiana.

\section{Raw materials}

Three varieties of paddy namely PR-115, PR-118 and Punjab Mehak were procured from Department of Plant Breeding and Genetics, PAU, Ludhiana.

\section{Milling}

Paddy was shelled to obtain brown rice in Satake Rice Sheller. For each milling test, paddy samples $(100 \mathrm{~g})$ were cleaned before passing through a Satake rubber roll huller (Model THU 35A, Japan). Broken rice was separated from head rice before packaging using a Satake grader (Model TRG05B, Japan) process.

\section{Hydrothermal treatments}

Paddy samples of all three varieties were parboiled by three different treatments listed below:

1. Soaking paddy in water at room temperature for 2 hours followed by steaming for 15 minutes under atmospheric pressure and drying by conventional and microwave method.

2. Soaking paddy in water at room temperature for 2 hours followed by steaming for 5-10 minutes under 15-20 PSI pressure and drying by conventional and microwave method.

3. Soaking paddy in hot water $\left(70 \pm 2^{\circ} \mathrm{C}\right)$ for $2-3$ hours followed by steaming for 15 minutes under atmospheric pressure and drying by conventional and microwave method.

For convenience and proper presentation of data, abbreviations have been used in the subsequent part of results and discussion. Details of these abbreviations were given in Table 1.a. 
Packaging: Brown rice packed in PET jars, cloth bags, plastic bags (HDPE) and vacuum packed in plastic bags and stored under room temperature for 4 months.

\section{Physicochemical characteristics of brown rice}

Moisture content: Weighed ground samples $(2 \mathrm{~g})$ were dried in hot air oven at $130^{\circ} \mathrm{C}$ for 1 hour and moisture content in percent was calculated from loss of weight (AACC 2000)

Thousand grain weight: Thousand kernel weight of head rice were counted and weighed accurately.

Bulk density: Kernels were poured in measuring cylinder up to fixed volume from fixed height mass of samples occupying fixed volume was measured. From ratio of mass to volume bulk density was calculated.

Length/Breadth ratio: For length (L) 10 grains were arranged in a straight line on a table and cumulative length was measured in $\mathrm{cm}$ with scale. Similarly for breadth (B) 10 kernels were arranged breadth wise and cumulative breadth was measured in $\mathrm{cm}$ with scale. The L/B value was calculated for brown rice for each treatment given to paddy.

Protein: Total nitrogen was determined by the macro-kjeldahl procedure (AACC 2000). One gram of sample was digested in kjeldahl flask with digestion mixture (copper sulphate and potassium sulphate in 1:9 ratios) and concentrated sulphuric acid $(20 \mathrm{ml})$ till light green color appeared and finally cooled. Ammonia released by distillation of digested samples with saturated $\mathrm{NaOH}(80 \mathrm{ml})$ was captured in $0.1 \mathrm{~N} \mathrm{HCL}$ and per cent $\mathrm{N}_{2}$ was estimated. The protein content was calculated as per cent nitrogen* factor. The 5.95 factor used for rice for calculations of protein $(\%)$.

Table 1a Description of experimental samples and the abbreviations used

\begin{tabular}{lc}
\hline Varieties (V) & Abbreviation \\
\hline PR 115 & $\mathrm{V}_{1}$ \\
\hline PR 118 & $\mathrm{V}_{2}$ \\
\hline Punjab Mehak & $\mathrm{V}_{3}$ \\
\hline Treatments (T) & Abbreviation \\
\hline Control & $\mathrm{T}_{1}$ \\
\hline $\begin{array}{l}\text { Soaking paddy in water at room temperature for 2 hours } \\
\text { followed by } \\
\text { steaming for 15 minutes under atmospheric pressure }\end{array}$ & $\mathrm{T}_{2}$ \\
\hline
\end{tabular}

Soaking paddy in water at room temperature for 2 hours

followed by

steaming for 5-10 minutes under 15-20 PSI

$\mathrm{T}_{3}$

Soaking paddy in hot water $\left(70 \pm 2^{\circ} \mathrm{C}\right)$ for $2-3$ hours followed by steaming for 15 minutes under atmospheric pressure

\begin{tabular}{lc}
\hline Packaging materials & Abbreviation \\
\hline Polythene packaging & $\mathrm{P}_{1}$ \\
\hline Jute/cloth bags & $\mathrm{P}_{2}$ \\
\hline Vacuum plastic bags & $\mathrm{P}_{3}$ \\
\hline PET jars & $\mathrm{P}_{4}$ \\
\hline Storage period (S) & Abbreviation \\
\hline 0 day & $\mathrm{S}_{1}$ \\
\hline I month & $\mathrm{S}_{2}$ \\
\hline 2 month & $\mathrm{S}_{3}$ \\
\hline 3 month & $\mathrm{S}_{4}$ \\
\hline 4 month & $\mathrm{S}$ \\
\hline Interactions & $\mathrm{Abbreviation}$ \\
\hline Variety $\times$ Treatment & $\mathrm{V} \times \mathrm{T}$ \\
\hline Treatments $\times$ Packaging & $\mathrm{T} \times \mathrm{P}$ \\
\hline Treatments $\times$ Storage period & $\mathrm{T} \times \mathrm{S}$ \\
\hline Variety $\times$ Packaging & $\mathrm{V} \times \mathrm{P}$ \\
\hline Variety $\times$ Storage period & $\mathrm{V} \times \mathrm{S}$ \\
\hline Packaging $\times$ Storage period & $\mathrm{P} \times \mathrm{S}$ \\
\hline
\end{tabular}

Fat: Fat analysis was done using Soxtech apparatus. Weighed sample (3g) was taken in thimble. Extraction cups were dried in oven at $130^{\circ} \mathrm{C}$ for $15 \mathrm{~min}$ and weight of empty cups were measured. After cooling $70 \mathrm{~m} 1$ of petroleum ether was added. When temperature was attained, the extraction cups were attached to the instrument and boiled for 30 minute and rinsed for 20 minutes. The recovered ether was collected and the fat contained in extraction cups was estimated.

Ash: Weighed ( $2 \mathrm{~g})$ sample was first incinerated on hot plate until there was no wore fumes comes out from samples then it was kept in muffle furnace at $550^{\circ} \mathrm{C}$ for 5 hours and results were expressed in per cent ash.

Amylose content: Very finely ground brown rice samples were sieved through a 100 mesh sieve. $100 \mathrm{mg}$ of each sample were weighed and taken in test tubes to which alcohol $(1 \mathrm{ml})$ and $1 \mathrm{~N} \mathrm{NaOH}(9 \mathrm{~m} 1)$ were added. The tubes were heated for 10 minutes in boiling water bath to gelatinize the starch, then cooled and transferred with several washings of water into a $100 \mathrm{ml}$ volumetric flask, brought into volume with water and mixed well. $5 \mathrm{ml}$ of starch solution was pipette for each sample in $100 \mathrm{ml}$ flasks. The color was developed by adding $1 \mathrm{~N}$ acetic acid $(1 \mathrm{ml})$ and iodine solution $(2 \mathrm{ml})$ according to the procedure developed by Juliano (1971). The solution was made up to volume, shaken and let stand for 20 minutes. Absorbance of solution was measured at $620 \mathrm{~nm}$. A standard curve was plotted by using potato amylose. Amylose content of samples was obtained by reference to the standard curve. The dilution factor of 20 for the sample was included in the conversion factor. The standard curve for amylose was given in Fig. 1.

Free fatty acids (FFA): Standard AOAC (2000) procedure was followed to estimate FFA. Weighed $5 \mathrm{~g}$ of sample was taken in flask. Add $50 \mathrm{ml}$ benzene and kept for $30 \mathrm{~min}$ for extraction of free fatty

acid. Took $5 \mathrm{ml}$ extract in flask, add $5 \mathrm{ml}$ benzene, $10 \mathrm{ml}$ alcohol and few drops of phenolphthalein as indicator and titrated against $0.02 \mathrm{~N} \mathrm{KOH}$ till light pink color disappeared.

$$
\% \mathrm{FFA}=\frac{282 \times 0.02 \mathrm{~N} \mathrm{KOH} \times \text { ml.of alkali used } \times \text { dilution factors }}{1000 \times \text { weight of sample taken }} \times 100
$$

\section{Peroxide value}

Standard AOAC (2000) procedure was followed to determine peroxide value of brown rice. Prepare acetic acid: chloroform solution in $2: 1$ ratio. $30 \mathrm{ml}$ of acetic acid: chloroform $(2: 1)$ was added to the weighed sample. It was kept under cool and dark place for $30 \mathrm{~min}$ and then $30 \mathrm{ml}$ of distilled water was added. The mixture was then shaken. This was slowly treated with $0.1 \mathrm{~N} \mathrm{Na}_{2} \mathrm{~S}_{2} \mathrm{O}_{3}$ vigorous shaking until yellow color disappeared. Then $0.5 \mathrm{ml}$ of 1.0 per cent starch solution was added and titrated continuously with vigorous shaking to release all iodine from chloroform layer until pink color just disappeared. The blank was run side by side. Peroxide value was determined by following formula:

value $(\mathrm{meq} / \mathrm{kg})$ Weight of formulation (g) $\times 100$

\section{Statistical analysis of data}

Data collected from aforesaid experiments was subjected to statistical analysis with the help of factorial design in CRD.

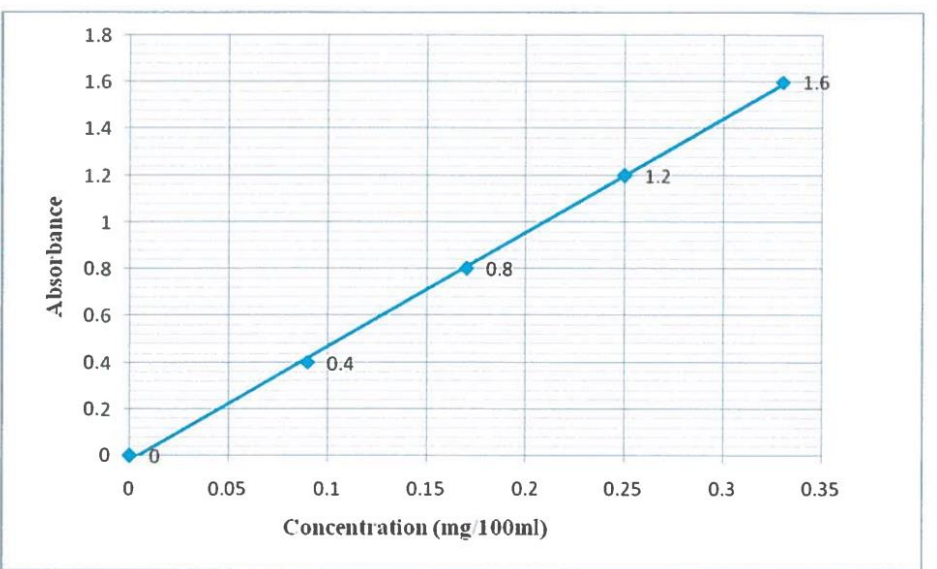

Figure 1 Standard curve for amylose

\section{Results and discussion}

\section{Physical parameters of raw paddy influenced by variety}

It was noticed that varieties show little variation in moisture content in raw paddy. Moisture content did not vary significantly in ease of raw paddy but PR 118 had higher moisture content as compared to PR 115 and Punjab Mehak varieties (Table 1.b). Varietal difference in percentage moisture content of paddy largely depends on ambient temperature and relative humidity of surroundings Varietal differences with regard to thousand kernel weight, bulk density, length, breadth and L/B ratio of paddy samples was found to be significant. Thousand kernel weights were higher for Punjab Mehak followed by PR-118 and least for 
PR-115. Data for bulk density showed significant differences among varieties showing higher bulk density for PR-115 and least for Punjab Mehak. The reason for 1ow bulk density for certain varieties might be due to that these varieties have long awns which were bulky and occupies space by keeping paddy grains apart such that it caused a reduction in the total mass per unit volume occupied by the grains (Alizadeh et al. 2006). The Bulk density of grain was used to design of silos and storage bins. Length was higher for Punjab Mehak and breadth was higher for PR-118. L/B ratio was higher for Punjab Mehak followed by PR-115, and PR-118, respectively. This showed that grains of Punjab Mehak were long and slender whereas PR-118 was short and bold.

Table 1b Physical parameters of raw paddy varieties

\begin{tabular}{lcccccc}
\hline Variety & $\begin{array}{c}\text { Moisture } \\
\text { content }(\boldsymbol{\%})\end{array}$ & $\begin{array}{c}\mathbf{1 0 0 0} \text {-kernel weight } \\
(\mathbf{g})\end{array}$ & Bulk density $(\mathbf{g} / \mathbf{m l})$ & Length $(\mathbf{c m})$ & Breadth $(\mathbf{c m})$ & L/B Ratio \\
\hline $\mathbf{V}_{\mathbf{1}}$ & $8.60 \pm 0.10$ & $22.32 \pm 0.03$ & $0.508 \pm 0.006$ & $9.3 \pm 0.10$ & $2.16 \pm 0.05$ & $\mathbf{4 . 2 9} \pm \mathbf{0 . 1 2}$ \\
\hline $\mathbf{V}_{\mathbf{2}}$ & $8.61 \pm 0.03$ & $24.15 \pm 0.02$ & $0.543 \pm 0.007$ & $8.83 \pm 0.05$ & $2.26 \pm 0.05$ & $\mathbf{3 . 8 9} \pm \mathbf{0 . 0 9}$ \\
\hline $\mathbf{V}_{\mathbf{3}}$ & $8.46 \pm 0.05$ & $25.42 \pm 0.03$ & $0.501 \pm 0.003$ & $10.76 \pm 0.05$ & $2.03 \pm 0.05$ & $\mathbf{5 . 2 9} \pm \mathbf{0 . 1 7}$ \\
\hline $\mathbf{C D}(\mathbf{p} \leq \mathbf{0 . 0 5})$ & $\mathbf{N S}$ & $\mathbf{0 . 6 4 9}$ & $\mathbf{0 . 1 2 1}$ & $\mathbf{0 . 1 4 8}$ & $\mathbf{0 . 1 1 5}$ & $\mathbf{0 . 2 7 2}$ \\
\hline
\end{tabular}

Milling quality of brown rice as influenced by variety and hydrothermal treatment

Table 2a Effect of treatments on milling quality of paddy

\begin{tabular}{cccc}
\hline Varieties & Treatment & Brown rice (\%) & $\begin{array}{c}\text { Brown head rice (on } \\
\text { \% brown rice) }\end{array}$ \\
\hline \multirow{3}{*}{$\mathrm{V}$} & $\mathrm{T}_{1}$ & $78.0 \pm 0.01$ & $81.15 \pm 0.025$ \\
\cline { 2 - 4 } & $\mathrm{T}_{2}$ & $79.2 \pm 0.03$ & $88.50 \pm 0.020$ \\
\cline { 2 - 4 } & $\mathrm{T}_{3}$ & $80.5 \pm 0.02$ & $94.70 \pm 0.020$ \\
\cline { 2 - 4 } & $\mathrm{T}_{4}$ & $80.1 \pm 0.01$ & $96.90 \pm 0.030$ \\
\hline \multirow{3}{*}{$\mathrm{V}$} & $\mathrm{T}_{1}$ & $79.7 \pm 0.01$ & $82.70 \pm 0.020$ \\
\cline { 2 - 4 } & $\mathrm{T}_{2}$ & $80.2 \pm 0.03$ & $88.90 \pm 0.025$ \\
\cline { 2 - 4 } & $\mathrm{T}_{3}$ & $80.3 \pm 0.02$ & $94.50 \pm 0.010$ \\
\cline { 2 - 4 } & $\mathrm{T}_{4}$ & $81.8 \pm 0.03$ & $97.10 \pm 0.010$ \\
\hline & $\mathrm{T}_{1}$ & $78.0 \pm 0.01$ & $82.50 \pm 0.035$ \\
\cline { 2 - 4 } & $\mathrm{T}_{2}$ & $80.0 \pm 0.01$ & $88.30 \pm 0.030$ \\
\cline { 2 - 4 } & $\mathrm{T}_{3}$ & $81.7 \pm 0.02$ & $94.90 \pm 0.020$ \\
\cline { 2 - 4 } & $\mathrm{T}_{4}$ & $81.1 \pm 0.02$ & $97.40 \pm 0.010$ \\
\hline $\mathrm{CD}$ & & $\mathrm{V}: 0.561, \mathrm{~T}: 0.18 ;$ & $\mathrm{V}: 1.67, \mathrm{~T}: 1.93 ;$ \\
$(\mathrm{p} \leq 0.05)$ & & $\mathrm{V} \times \mathrm{T}: 1.23$ & $\mathrm{~V} \times \mathrm{T}: 3.35$ \\
\hline
\end{tabular}

Milling quality was assessed by determining percentages of brown rice and head rice (on per cent brown rice). Varieties PR-115, PR-118 and Punjab Mehak differed significantly for brown rice (\%) and head rice (\%). Treatments and variety were found to had significant effect on brown rice out-turns; treatments increase the brown rice out-turn but to negligible level (Table 2.a). Interaction of varieties with treatments was found to be significant for both brown rice (\%) and brown head rice $(\%)$. Data on per cent head rice recoveries showed significant differences for varieties and treatments. Punjab Mehak gave higher percentages of head rice followed by PR-118 and lowest for PR-115. Treatments increased per cent head rice recoveries significantly over their respective controls. Treatment had the effect of increasing head rice recoveries making $\mathrm{T}_{4}$ best in yielding higher head rice followed by $T_{3}$ and $T_{2}$ respectively over the control $\left(T_{1}\right)$. Significant interaction was observed for varieties with treatments.

Sareepuang et al. (2008) found that head rice yield was significantly increased from 51 per cent in brown rice to 59,83 and 84 per cent in PR-40, PR-50 and PR-60, respectively. Saif et al. (2004) reported that the increase in length, width and thickness due to parboiling process, leading to some advantages over the unparboiled one such as the strengthening of kernel integrity, increase of milling recovery and decrease of cooking losses.

\section{Chemical composition and pasting properties of brown rice}

Brown rice were analyzed for chemical composition and pasting profile to know the different concentration of parameters in all three varieties (Table 2.b) of brown rice after milling. PR-115 had $8.38 \%$ protein, $2.25 \%$ fat, $0.95 \%$ ash, $0.27 \% \mathrm{FFA}, 0.79 \mathrm{meq} / \mathrm{kg}$ peroxide value, $19.85 \%$ amylase, $92.40^{\circ} \mathrm{C}$ pasting temperature, $2415 \mathrm{cP}$ peak viscosity, $1051 \mathrm{cP}$ hold viscosity, $2826 \mathrm{cP}$ final viscosity, $667 \mathrm{cP}$ breakdown viscosity and $741 \mathrm{cP}$ setback viscosity. PR-118 had $8.31 \%$ protein, $2.98 \%$ fat, $0.90 \%$ ash, $0.22 \%$ FFA, 0.79 meq $/ \mathrm{kg}$ peroxide value, $23.04 \%$ amylase, $85.70^{\circ} \mathrm{C}$ pasting temperature, $2456 \mathrm{cP}$ peak viscosity, $1331 \mathrm{cP}$ hold viscosity, $2736 \mathrm{cP}$ final viscosity, $445 \mathrm{cP}$ breakdown viscosity and $981 \mathrm{cP}$ setback viscosity. Punjab Mehak $8.15 \%$ protein, $2.96 \%$ fat, $1.05 \%$ ash, $0.25 \%$ FFA, $0.78 \mathrm{meq} / \mathrm{kg}$ peroxide value, $22.08 \%$ amylase, $87.50^{\circ} \mathrm{C}$ pasting temperature, $2345 \mathrm{cP}$ peak viscosity, $1431 \mathrm{cP}$ hold viscosity, $2500 \mathrm{cP}$ final viscosity, $450 \mathrm{cP}$ breakdown viscosity and $1200 \mathrm{cP}$ setback viscosity.

Table $2 \mathbf{b}$ Chemical composition and pasting properties of brown rice

\begin{tabular}{lccc}
\hline \multirow{2}{*}{ Composition } & PR-115 & Varieties & Punjab Mehak \\
\cline { 2 - 4 } & $8.38 \pm 0.01$ & $8.31 \pm 0.02$ & $8.15 \pm 0.02$ \\
\hline Protein $(\%)$ & $2.25 \pm 0.01$ & $2.98 \pm 0.03$ & $2.96 \pm 0.02$ \\
\hline Fat $(\%)$ & $0.95 \pm 0.02$ & $0.90 \pm 0.03$ & $1.05 \pm 0.04$ \\
\hline Ash $(\%)$ & $0.27 \pm 0.03$ & $0.22 \pm 0.01$ & $0.25 \pm 0.02$ \\
\hline Free fatty acid (\% as oleic acid) & $0.79 \pm 0.03$ & $0.79 \pm 0.03$ & $0.78 \pm 0.03$ \\
\hline Peroxide value $(\mathrm{meq} / \mathrm{kg})$ & $19.85 \pm 1.65$ & $23.04 \pm 3.12$ & $22.08 \pm 43.08$ \\
\hline Amylose $(\%)$ & $92.40 \pm 1.74$ & $85.70 \pm 0.44$ & $87.50 \pm 1.65$ \\
\hline Pasting temperature $\left({ }^{\circ} \mathrm{C}\right)$ & $2415 \pm 2.11$ & $2456 \pm 3.51$ & $2345 \pm 6.14$ \\
\hline Peak viscosity $(\mathrm{cP})$ & $1051 \pm 3.12$ & $1331 \pm 3.31$ & $1431 \pm 1.08$ \\
\hline Hold viscosity $(\mathrm{cP})$ & $2826 \pm 6.15$ & $2736 \pm 4.75$ & $2500 \pm 3.48$ \\
\hline Final viscosity $(\mathrm{cP})$ & $667 \pm 2.12$ & $445 \pm 2.36$ & $450 \pm 1.54$ \\
\hline Breakdown $(\mathrm{cP})$ & $741 \pm 2.25$ & $981 \pm 2.21$ & $1200 \pm 2.56$ \\
\hline Set back $(\mathrm{cP})$ & &
\end{tabular}

Physical parameters of brown rice as influenced by treatments, packaging and storage

\section{Effect of variety, treatments, packaging material and storage period on moisture content (\%) of brown rice}

There was a significant difference in moisture content with respect to varieties, treatments and storage period however there was found that storage period had insignificant impact on the moisture content of parboiled brown rice (Table 3.a) The interaction of treatments with varieties was found to be significant effect. Changes in moisture content of brown rice due to treatment largely depend on ambient temperature, relative humidity and method of treatments. Hydrothermal treatments demonstrated a non significant effect on moisture content of brown, milled rice and bran of all varieties as a result no significant difference was observed. Among the varieties Punjab Mehak had maximum moisture content after the hydrothermal treatment as compared to PR-115 and PR-118. The individual effect of variety, treatment, storage period was found to be significant while packaging material had insignificant effect on moisture content of parboiled brown rice. The interaction of varieties with treatment had significant effect while interaction of varieties with storage period, treatments with storage period and packaging materials with storage period had insignificant effect on moisture content of parboiled brown rice. The moisture content decreased with increased storage periods. Less moisture content was observed in all fractions in both raw and parboiled varieties, reason was that before milling paddy was dried 
up to 9 per cent moisture level and during milling it was again reduced (Farhan 2011).

Table 3a Effect of variety, treatments, packaging material and storage period on moisture content (\%) of brown rice

\begin{tabular}{|c|c|c|c|c|c|c|c|}
\hline \multirow{2}{*}{ Variety } & \multirow{2}{*}{ Treatment } & \multirow{2}{*}{$\begin{array}{l}\text { Packaging } \\
\text { Material }\end{array}$} & \multicolumn{5}{|c|}{ Storage Period } \\
\hline & & & $S_{1}$ & $\mathbf{S}_{2}$ & $\mathbf{S}_{3}$ & $S_{4}$ & $S_{5}$ \\
\hline \multirow{16}{*}{$\mathrm{V}_{1}$} & \multirow{4}{*}{$\mathrm{T}_{1}$} & $\mathrm{P}_{1}$ & $11.020 \pm 0.02$ & $11.010 \pm .010$ & $11.090 \pm 0.25$ & $11.110 \pm 0.01$ & $11.240 \pm 0.02$ \\
\hline & & $\mathrm{P}_{2}$ & $11.020 \pm 0.02$ & $11.600 \pm .010$ & $11.800 \pm 0.03$ & $12.000 \pm 0.02$ & $12.500 \pm 0.02$ \\
\hline & & $\mathrm{P}_{3}$ & $11.020 \pm 0.02$ & $11.020 \pm 0.02$ & $11.021 \pm 0.03$ & $11.022 \pm 0.01$ & $11.023 \pm 0.03$ \\
\hline & & $\mathrm{P}_{4}$ & $11.020 \pm 0.02$ & $11.021 \pm 0.02$ & $11.022 \pm 0.02$ & $11.030 \pm 0.01$ & $11.034 \pm 0.03$ \\
\hline & \multirow{4}{*}{$\mathrm{T}_{2}$} & $\mathrm{P}_{1}$ & $12.210 \pm 0.16$ & $12.220 \pm 0.02$ & $12.220 \pm 0.01$ & $12.210 \pm 0.02$ & $12.230 \pm 0.01$ \\
\hline & & $\mathrm{P}_{2}$ & $12.210 \pm 0.16$ & $12.600 \pm 0.03$ & $12.800 \pm 0.01$ & $13.000 \pm 0.03$ & $13.500 \pm 0.02$ \\
\hline & & $\mathrm{P}_{3}$ & $12.210 \pm 0.16$ & $12.210 \pm 0.02$ & $12.200 \pm 0.01$ & $12.190 \pm 0.02$ & $12.190 \pm 0.01$ \\
\hline & & $\mathrm{P}_{4}$ & $12.210 \pm 0.16$ & $12.210 \pm 0.01$ & $12.220 \pm 0.01$ & $12.221 \pm 0.02$ & $12.225 \pm 0.02$ \\
\hline & \multirow{4}{*}{$\mathrm{T}_{3}$} & $\mathrm{P}_{1}$ & $12.900 \pm 0.04$ & $13.000 \pm 0.04$ & $13.010 \pm 0.02$ & $13.010 \pm 0.03$ & $13.020 \pm 0.01$ \\
\hline & & $\mathrm{P}_{2}$ & $12.900 \pm 0.04$ & $13.200 \pm 0.04$ & $13.700 \pm 0.03$ & $13.900 \pm 0.04$ & $14.200 \pm 0.12$ \\
\hline & & $\mathrm{P}_{3}$ & $12.900 \pm 0.04$ & $12.900 \pm 0.05$ & $12.890 \pm 0.01$ & $12.880 \pm 0.04$ & $12.885 \pm 0.13$ \\
\hline & & $\mathrm{P}_{4}$ & $12.900 \pm 0.04$ & $12.920 \pm 0.01$ & $12.930 \pm 0.01$ & $12.935 \pm 0.05$ & $12.939 \pm 0.12$ \\
\hline & \multirow{4}{*}{$\mathrm{T}_{4}$} & $\mathrm{P}_{1}$ & $12.540 \pm 0.15$ & $12.550 \pm 0.01$ & $12.530 \pm 0.03$ & $12.530 \pm 0.01$ & $12.520 \pm 0.15$ \\
\hline & & $\mathrm{P}_{2}$ & $12.540 \pm 0.15$ & $13.300 \pm 0.03$ & $13.600 \pm 0.05$ & $13.900 \pm 0.01$ & $14.100 \pm 0.21$ \\
\hline & & $\mathrm{P}_{3}$ & $12.540 \pm 0.15$ & $12.540 \pm 0.12$ & $12.539 \pm 0.04$ & $12.535 \pm 0.02$ & $12.490 \pm 0.24$ \\
\hline & & $\mathrm{P}_{4}$ & $12.540 \pm 0.15$ & $12.610 \pm 0.05$ & $12.620 \pm 0.04$ & $12.630 \pm 0.03$ & $12.630 \pm 0.15$ \\
\hline \multirow{16}{*}{$\mathrm{V}_{2}$} & \multirow{4}{*}{$\mathrm{T}_{1}$} & $\mathrm{P}_{1}$ & $11.610 \pm 0.01$ & $11.620 \pm 0.14$ & $11.690 \pm 0.01$ & $12.050 \pm 0.01$ & $12.080 \pm 0.02$ \\
\hline & & $\mathrm{P}_{2}$ & $11.610 \pm 0.01$ & $11.900 \pm 0.13$ & $12.000 \pm 0.01$ & $12.400 \pm 0.13$ & $13.000 \pm 0.03$ \\
\hline & & $\mathrm{P}_{3}$ & $11.610 \pm 0.01$ & $11.600 \pm 0.12$ & $11.590 \pm 0.02$ & $11.599 \pm 0.12$ & $11.599 \pm 0.02$ \\
\hline & & $\mathrm{P}_{4}$ & $11.610 \pm 0.01$ & $11.610 \pm 0.02$ & $11.612 \pm 0.02$ & $11.613 \pm 0.02$ & I $1.620 \pm 0.02$ \\
\hline & \multirow{4}{*}{$\mathrm{T}_{2}$} & $\mathrm{P}_{1}$ & $12.430 \pm 0.03$ & $12.440 \pm 0.03$ & $12.430 \pm 0.03$ & $12.450 \pm 0.13$ & $12.420 \pm 0.02$ \\
\hline & & $\mathrm{P}_{2}$ & $12.430 \pm 0.03$ & $12.800 \pm 0.02$ & $12.900 \pm 0.02$ & $13.200 \pm 0.15$ & $13.600 \pm 0.01$ \\
\hline & & $\mathrm{P}_{3}$ & $12.430 \pm 0.03$ & $12.429 \pm 0.01$ & $12.428 \pm 0.01$ & $12.427 \pm 0.16$ & $12.427 \pm 0.02$ \\
\hline & & $\mathrm{P}_{4}$ & $12.430 \pm 0.03$ & $12.430 \pm 0.01$ & $12.435 \pm 0.01$ & $12.440 \pm 0.12$ & $12.445 \pm 0.01$ \\
\hline & \multirow{4}{*}{$\mathrm{T}_{3}$} & $\mathrm{P}_{1}$ & $13.000 \pm 0.13$ & $13.020 \pm 0.01$ & $13.010 \pm 0.01$ & $13.020 \pm 0.02$ & $13.020 \pm 0.03$ \\
\hline & & $\mathrm{P}_{2}$ & $13.000 \pm 0.13$ & $13.500 \pm 0.01$ & $13.600 \pm 0.02$ & $13.900 \pm 0.03$ & $14.500 \pm 0.05$ \\
\hline & & $\mathrm{P}_{3}$ & $13.000 \pm 0.13$ & $13.000 \pm 0.02$ & $12.990 \pm 0.02$ & $12.990 \pm 0.14$ & $12.980 \pm 0.04$ \\
\hline & & $\mathrm{P}_{4}$ & $13.000 \pm 0.13$ & $13.000 \pm 0.25$ & $13.100 \pm 0.03$ & $13.100 \pm 0.01$ & $13.200 \pm 0.01$ \\
\hline & \multirow{4}{*}{$\mathrm{T}_{4}$} & $\mathrm{P}_{1}$ & $12.890 \pm 0.02$ & $12.890 \pm 0.01$ & $12.880 \pm 0.03$ & $12.900 \pm 0.02$ & $12.880 \pm 0.02$ \\
\hline & & $\mathrm{P}_{2}$ & $12.890 \pm 0.02$ & $13.200 \pm 0.01$ & $13.500 \pm 0.05$ & $13.900 \pm 0.02$ & $14.600 \pm 0.02$ \\
\hline & & $\mathrm{P}_{3}$ & $12.890 \pm 0.02$ & $12.880 \pm 0.02$ & $12.880 \pm 0.05$ & $12.870 \pm 0.03$ & $12.870 \pm 0.01$ \\
\hline & & $\mathrm{P}_{4}$ & $12.890 \pm 0.02$ & $12.900 \pm 0.06$ & $12.900 \pm 0.05$ & $12.920 \pm 0.02$ & $12.970 \pm 0.03$ \\
\hline \multirow{16}{*}{$\mathrm{V}_{3}$} & \multirow{4}{*}{$\mathrm{T}_{1}$} & $\mathrm{P}_{1}$ & $10.080 \pm 0.02$ & $10.000 \pm 0.04$ & $10.120 \pm 0.04$ & $10.130 \pm 0.01$ & $10.130 \pm 0.01$ \\
\hline & & $\mathrm{P}_{2}$ & $10.080 \pm 0.02$ & $9.900 \pm 0.01$ & $10.600 \pm 0.05$ & $10.900 \pm 0.01$ & $11.200 \pm 0.02$ \\
\hline & & $\mathrm{P}_{3}$ & $10.080 \pm 0.02$ & $10.079 \pm 0.04$ & $10.079 \pm 0.04$ & $10.075 \pm 0.01$ & $10.076 \pm 0.02$ \\
\hline & & $\mathrm{P}_{4}$ & $10.080 \pm 0.02$ & $10.081 \pm 0.02$ & $10.082 \pm 0.06$ & $10.082 \pm 0.02$ & $10.085 \pm 0.03$ \\
\hline & \multirow{4}{*}{$\mathrm{T}_{2}$} & $\mathrm{P}_{1}$ & $13.850 \pm 0.15$ & $13.830 \pm 0.02$ & $13.520 \pm 0.01$ & $13.820 \pm 0.03$ & $13.830 \pm 0.01$ \\
\hline & & $\mathrm{P}_{2}$ & $13.850 \pm 0.15$ & $13.800 \pm 0.03$ & $13.500 \pm 0.01$ & $13.900 \pm 0.04$ & $14.000 \pm 0.01$ \\
\hline & & $\mathrm{P}_{3}$ & $13.850 \pm 0.15$ & $13.848 \pm 0.01$ & $13.845 \pm 0.02$ & $13.845 \pm 0.04$ & $13.841 \pm 0.04$ \\
\hline & & $\mathrm{P}_{4}$ & $13.850 \pm 0.15$ & $13.855 \pm 0.02$ & $13.859 \pm 0.02$ & $13.859 \pm 0.05$ & $13.860 \pm 0.04$ \\
\hline & \multirow{4}{*}{$\mathrm{T}_{3}$} & $\mathrm{P}_{1}$ & $13.050 \pm 0.01$ & $13.060 \pm 0.05$ & $13.060 \pm 0.12$ & $13.050 \pm 0.01$ & $13.040 \pm 0.02$ \\
\hline & & $\mathrm{P}_{2}$ & $13.050 \pm 0.01$ & $13.300 \pm 0.05$ & $13.600 \pm 0.12$ & $13.700 \pm 0.02$ & $14.300 \pm 0.03$ \\
\hline & & $\mathrm{P}_{3}$ & $13.050 \pm 0.01$ & $13.050 \pm 0.01$ & $13.040 \pm 0.13$ & $13.010 \pm 0.03$ & $13.010 \pm 0.02$ \\
\hline & & $\mathrm{P}_{4}$ & $13.050 \pm 0.01$ & $13.060 \pm 0.02$ & $13.060 \pm 0.13$ & $13.068 \pm 0.01$ & $13.090 \pm 0.01$ \\
\hline & \multirow{4}{*}{$\mathrm{T}_{4}$} & $\mathrm{P}_{1}$ & $13.010 \pm 0.03$ & $13.010 \pm 0.01$ & $13.020 \pm 0.13$ & $13.020 \pm 0.03$ & $13.010 \pm 0.02$ \\
\hline & & $\mathrm{P}_{2}$ & $13.010 \pm 0.03$ & $12.900 \pm 0.01$ & $13.000 \pm 0.12$ & $13.300 \pm 0.01$ & $13.900 \pm 0.01$ \\
\hline & & $\mathrm{P}_{3}$ & $13.010 \pm 0.03$ & $13.000 \pm 0.04$ & $13.010 \pm 0.02$ & $12.990 \pm 0.01$ & $12.980 \pm 0.01$ \\
\hline & & $\mathrm{P}_{4}$ & $13.010 \pm 0.03$ & $13.010 \pm 0.03$ & $13.020 \pm 0.02$ & $13.022 \pm 0.13$ & $13.030 \pm 0.03$ \\
\hline
\end{tabular}

$\mathrm{CD}(\mathrm{p} \leq 0.05) \mathrm{V}: 0.29, \mathrm{~T}: 0.34, \mathrm{~S}: 0.59, \mathrm{P}: \mathrm{NS}, \mathrm{V} \times \mathrm{T}: 0.33, \mathrm{~V} \times \mathrm{S}: \mathrm{NS}, \mathrm{T} \times \mathrm{S}: \mathrm{NS}, \mathrm{P} \times \mathrm{S}: \mathrm{NS}$

Effect of variety, treatments, packaging material and storage period on $\mathrm{L} / \mathrm{B}$ ratio of brown rice

Varieties, treatments and storage had a significant effect on L/B ratio, whereas packaging material showed insignificant on $\mathrm{L} / \mathrm{B}$ ratio of parboiled brown rice. $\mathrm{V}_{3}$ had maximum $\mathrm{L} / \mathrm{B}$ ratio followed by $\mathrm{V}_{2}$ and $\mathrm{V}_{1}$ respectively (Table 3.b). Among the varieties Punjab Mehak had maximum L/B ratio after the hydrothermal treatment as compared to PR-115 and PR-118. The interaction of varieties with treatment had significant effect while interaction of varieties with storage period, treatments with storage period and packaging materials with storage period had no significant effect on L/B ratio of parboiled brown rice. The parboiled milled brown rice obtained from Punjab Mehak with treatment $\mathrm{T}_{4}$ had maximum L/B ratio among the all treatments and other varieties. Farhan (2011) reported that $\mathrm{L} / \mathrm{B}$ ratio of basmati and coarse varieties were significantly differing from each other. Brown and milled rice fractions of Basmati varieties possessed more length breadth ratio in comparison with fractions of coarse varieties in both raw and parboiled varieties and there was no significant difference was found after parboiling.

Table 3b Effect of variety, treatments, packaging material and storage period on L/B ratio of brown rice 


\begin{tabular}{|c|c|c|c|c|c|c|c|}
\hline \multirow{2}{*}{ Variety } & \multirow{2}{*}{ Treatment } & \multirow{2}{*}{$\begin{array}{c}\text { Packaging } \\
\text { Material }\end{array}$} & \multicolumn{5}{|c|}{ Storage Period } \\
\hline & & & $\mathbf{S}_{1}$ & $\mathbf{S}_{2}$ & $\mathbf{S}_{3}$ & $\mathbf{S}_{4}$ & $S_{5}$ \\
\hline \multirow{16}{*}{$\mathbf{V}_{1}$} & \multirow{4}{*}{$\mathrm{T}_{1}$} & $\mathrm{P}_{1}$ & $3.490 \pm 0.01$ & $3.499 \pm 0.025$ & $3.492 \pm 0.012$ & $3.493 \pm 0.002$ & $3.495 \pm 0.003$ \\
\hline & & $\mathrm{P}_{2}$ & $3.770 \pm 0.01$ & $3.770 \pm 0.024$ & $3.670 \pm 0.012$ & $3.690 \pm 0.025$ & $3.660 \pm 0.012$ \\
\hline & & $\mathrm{P}_{3}$ & $3.770 \pm 0.01$ & $3.690 \pm 0.031$ & $3.670 \pm 0.013$ & $3.777 \pm 0.014$ & $3.660 \pm 0.031$ \\
\hline & & $\mathrm{P}_{4}$ & $3.770 \pm 0.01$ & $3.770 \pm 0.021$ & $3.660 \pm 0.014$ & $3.777 \pm 0.024$ & $3.770 \pm 0.021$ \\
\hline & \multirow{4}{*}{$\mathrm{T}_{2}$} & $\mathrm{P}_{1}$ & $3.660 \pm 0.03$ & $3.660 \pm 0.031$ & $3.670 \pm 0.015$ & $3.670 \pm 0.026$ & $3.661 \pm 0.021$ \\
\hline & & $\mathrm{P}_{2}$ & $3.660 \pm 0.03$ & $3.523 \pm 0.032$ & $3.541 \pm 0.014$ & $3.722 \pm 0.001$ & $3.770 \pm 0.005$ \\
\hline & & $\mathrm{P}_{3}$ & $3.660 \pm 0.03$ & $3.722 \pm 0.032$ & $3.541 \pm 0.025$ & $3.722 \pm 0.006$ & $3.770 \pm 0.004$ \\
\hline & & $\mathrm{P}_{4}$ & $3.660 \pm 0.03$ & $3.660 \pm 0.012$ & $3.770 \pm 0.026$ & $3.722 \pm 0.004$ & $3.523 \pm 0.006$ \\
\hline & \multirow{4}{*}{$\mathrm{T}_{3}$} & $\mathrm{P}_{1}$ & $3.640 \pm 0.01$ & $3.630 \pm 0.012$ & $3.640 \pm 0.023$ & $3.630 \pm 0.007$ & $3.634 \pm 0.014$ \\
\hline & & $\mathrm{P}_{2}$ & $3.630 \pm 0.01$ & $3.882 \pm 0.034$ & $3.880 \pm 0.021$ & $3.910 \pm 0.003$ & $3.954 \pm 0.021$ \\
\hline & & $\mathrm{P}_{3}$ & $3.630 \pm 0.01$ & $3.910 \pm 0.032$ & $3.880 \pm 0.025$ & $3.941 \pm 0.004$ & $3.954 \pm 0.034$ \\
\hline & & $\mathrm{P}_{4}$ & $3.630 \pm 0.01$ & $3.630 \pm 0.021$ & $3.954 \pm 0.025$ & $3.941 \pm 0.002$ & $3.882 \pm 0.054$ \\
\hline & \multirow{4}{*}{$\mathrm{T}_{4}$} & $\mathrm{P}_{1}$ & $3.550 \pm 0.02$ & $3.556 \pm 0.021$ & $3.555 \pm 0.021$ & $3.555 \pm 0.001$ & $3.554 \pm 0.064$ \\
\hline & & $\mathrm{P}_{2}$ & $3.550 \pm 0.02$ & $3.364 \pm 0.021$ & $3.550 \pm 0.026$ & $3.555 \pm 0.011$ & $3.530 \pm 0.061$ \\
\hline & & $\mathrm{P}_{3}$ & $3.550 \pm 0.02$ & $3.555 \pm 0.031$ & $3.550 \pm 0.028$ & $3.555 \pm 0.002$ & $3.530 \pm 0.061$ \\
\hline & & $\mathrm{P}_{4}$ & $3.550 \pm 0.02$ & $3.550 \pm 0.036$ & $3.530 \pm 0.004$ & $3.555 \pm 0.001$ & $3.364 \pm 0.021$ \\
\hline \multirow{16}{*}{$\mathbf{V}_{2}$} & \multirow{4}{*}{$\mathrm{T}_{1}$} & $\mathrm{P}_{1}$ & $3.300 \pm 0.03$ & $3.340 \pm 0.002$ & $3.330 \pm 0.005$ & $3.330 \pm 0.024$ & $3.340 \pm 0.001$ \\
\hline & & $\mathrm{P}_{2}$ & $3.300 \pm 0.03$ & $3.340 \pm 0.001$ & $3.500 \pm 0.003$ & $3.400 \pm 0.024$ & $3.400 \pm 0.021$ \\
\hline & & $\mathrm{P}_{3}$ & $3.300 \pm 0.03$ & $3.400 \pm 0.003$ & $3.500 \pm 0.001$ & $3.300 \pm 0.251$ & $3.400 \pm 0.021$ \\
\hline & & $\mathrm{P}_{4}$ & $3.300 \pm 0.03$ & $3.300 \pm 0.002$ & $3.400 \pm 0.001$ & $3.300 \pm 0.023$ & $3.340 \pm 0.003$ \\
\hline & \multirow{4}{*}{$\mathrm{T}_{2}$} & $P_{1}$ & $3.520 \pm 0.01$ & $3.521 \pm 0.015$ & $3.522 \pm 0.154$ & $3.523 \pm 0.008$ & $3.522 \pm 0.004$ \\
\hline & & $\mathrm{P}_{2}$ & $3.520 \pm 0.01$ & $3.623 \pm 0.014$ & $3.660 \pm 0.021$ & $3.621 \pm 0.004$ & $3.640 \pm 0.004$ \\
\hline & & $\mathrm{P}_{3}$ & $3.520 \pm 0.01$ & $3.621 \pm 0.014$ & $3.660 \pm 0.021$ & $3.611 \pm 0.003$ & $3.640 \pm 0.002$ \\
\hline & & $\mathrm{P}_{4}$ & $3.520 \pm 0.01$ & $3.520 \pm 0.016$ & $3.640 \pm 0.026$ & $3.611 \pm 0.003$ & $3.623 \pm 0.002$ \\
\hline & \multirow{4}{*}{$\mathrm{T}_{3}$} & $\mathrm{P}_{1}$ & $3.721 \pm 0.12$ & $3.728 \pm 0.012$ & $3.720 \pm 0.014$ & $3.723 \pm 0.012$ & $3.723 \pm 0.014$ \\
\hline & & $\mathrm{P}_{2}$ & $3.250 \pm 0.12$ & $3.728 \pm 0.014$ & $3.710 \pm 0.014$ & $3.560 \pm 0.012$ & $3.657 \pm 0.023$ \\
\hline & & $\mathrm{P}_{3}$ & $3.250 \pm 0.12$ & $3.560 \pm 0.014$ & $3.710 \pm 0.012$ & $3.578 \pm 0.021$ & $3.657 \pm 0.041$ \\
\hline & & $\mathrm{P}_{4}$ & $3.250 \pm 0.12$ & $3.250 \pm 0.012$ & $3.657 \pm 0.016$ & $3.578 \pm 0.001$ & $3.728 \pm 0.014$ \\
\hline & \multirow{4}{*}{$\mathrm{T}_{4}$} & $\mathrm{P}_{1}$ & $3.611 \pm 0.01$ & $3.612 \pm 0.012$ & $3.611 \pm 0.014$ & $3.611 \pm 0.001$ & $3.612 \pm 0.024$ \\
\hline & & $\mathrm{P}_{2}$ & $3.250 \pm 0.01$ & $3.460 \pm 0.013$ & $3.420 \pm 0.023$ & $3.490 \pm 0.002$ & $3.430 \pm 0.071$ \\
\hline & & $\mathrm{P}_{3}$ & $3.250 \pm 0.01$ & $3.490 \pm 0.012$ & $3.420 \pm 0.023$ & $3.611 \pm 0.003$ & $3.430 \pm 0.024$ \\
\hline & & $\mathrm{P}_{4}$ & $3.250 \pm 0.01$ & $3.250 \pm 0.011$ & $3.430 \pm 0.022$ & $3.611 \pm 0.012$ & $3.460 \pm 0.025$ \\
\hline \multirow{16}{*}{$\mathbf{V}_{3}$} & \multirow{4}{*}{$\mathrm{T}_{1}$} & $\mathrm{P}_{1}$ & $4.820 \pm 0.01$ & $4.840 \pm 0.012$ & $4.850 \pm 0.011$ & $4.823 \pm 0.001$ & $4.840 \pm 0.028$ \\
\hline & & $\mathrm{P}_{2}$ & $4.820 \pm 0.01$ & $4.840 \pm 0.012$ & $4.850 \pm 0.033$ & $4.830 \pm 0.002$ & $4.810 \pm 0.027$ \\
\hline & & $\mathrm{P}_{3}$ & $4.820 \pm 0.01$ & $4.830 \pm 0.014$ & $4.850 \pm 0.011$ & $4.823 \pm 0.001$ & $4.810 \pm 0.014$ \\
\hline & & $\mathrm{P}_{4}$ & $4.820 \pm 0.01$ & $4.820 \pm 0.024$ & $4.810 \pm 0.015$ & $4.823 \pm 0.025$ & $4.840 \pm 0.028$ \\
\hline & \multirow{4}{*}{$\mathrm{T}_{2}$} & $\mathrm{P}_{1}$ & $4.220 \pm 0.03$ & $4.221 \pm 0.014$ & $4.221 \pm 0.016$ & $4.222 \pm 0.026$ & $4.223 \pm 0.037$ \\
\hline & & $\mathrm{P}_{2}$ & $4.220 \pm 0.03$ & $4.587 \pm 0.021$ & $4.534 \pm 0.014$ & $4.761 \pm 0.014$ & $4.550 \pm 0.064$ \\
\hline & & $\mathrm{P}_{3}$ & $4.220 \pm 0.03$ & $4.761 \pm 0.024$ & $4.534 \pm 0.016$ & $4.764 \pm 0.012$ & $4.550 \pm 0.027$ \\
\hline & & $\mathrm{P}_{4}$ & $4.220 \pm 0.03$ & $4.220 \pm 0.016$ & $4.550 \pm 0.016$ & $4.764 \pm 0.045$ & $4.587 \pm 0.025$ \\
\hline & \multirow{4}{*}{$\mathrm{T}_{3}$} & $\mathrm{P}_{1}$ & $4.310 \pm 0.01$ & $4.310 \pm 0.014$ & $4.312 \pm 0.023$ & $4.313 \pm 0.054$ & $4.310 \pm 0.021$ \\
\hline & & $\mathrm{P}_{2}$ & $4.310 \pm 0.01$ & $4.642 \pm 0.014$ & $4.450 \pm 0.024$ & $4.490 \pm 0.014$ & $4.490 \pm 0.026$ \\
\hline & & $\mathrm{P}_{3}$ & $4.310 \pm 0.01$ & $4.490 \pm 0.017$ & $4.450 \pm 0.012$ & $4.823 \pm 0.024$ & $4.490 \pm 0.012$ \\
\hline & & $\mathrm{P}_{4}$ & $4.310 \pm 0.01$ & $4.310 \pm 0.015$ & $4.490 \pm 0.021$ & $4.823 \pm 0.024$ & $4.642 \pm 0.023$ \\
\hline & \multirow{4}{*}{$\mathrm{T}_{4}$} & $\mathrm{P}_{1}$ & $4.530 \pm 0.02$ & $4.531 \pm 0.031$ & $4.532 \pm 0.023$ & $4.531 \pm 0.026$ & $4.532 \pm 0.026$ \\
\hline & & $\mathrm{P}_{2}$ & $4.530 \pm 0.02$ & $4.500 \pm 0.015$ & $4.400 \pm 0.021$ & $4.530 \pm 0.031$ & $4.550 \pm 0.025$ \\
\hline & & $\mathrm{P}_{3}$ & $4.530 \pm 0.02$ & $4.530 \pm 0.013$ & $4.400 \pm 0.014$ & $4.764 \pm 0.031$ & $4.550 \pm 0.004$ \\
\hline & & $\mathrm{P}_{4}$ & $4.530 \pm 0.02$ & $4.530 \pm 0.012$ & $4.550 \pm 0.014$ & $4.764 \pm 0.021$ & $4.500 \pm 0.005$ \\
\hline
\end{tabular}

Effect of variety, treatments, packaging material and storage period on 1000 kernel weight (g) of parboiled brown rice

The individual effect of varieties, treatments and storage period was significan while individual effect of packaging material was insignificant on 1000 kerne weight of parboiled brown rice. The interactions of treatment with varieties, treatment with storage period, varieties with storage period and packaging material with storage period were found significant on 1000 kernel weight of parboiled brown rice (Table 4.a). The thousand kernel weight is a measuremen of the net weight in grams of 1000 rice grain. The weight varies from one variety to another variety. Hydrothermal treatments demonstrated a significant effect on 1000 kernel weight $(\mathrm{g})$ of parboiled brown rice. Among the varieties Punjab Mehak had maximum thousand kernel weight after the hydrothermal treatment as compared to PR-118 and PR-115. The thousand kernel weight decreased with increased storage periods due to loss of moisture content. The thousand kernel weight of Basmati varieties was significantly lower than coarse varieties for both brown and milled rice grains of raw and parboiled rice (Farhan 2011). 


\begin{tabular}{|c|c|c|c|c|c|c|c|}
\hline \multirow[t]{2}{*}{ Variety } & \multirow[t]{2}{*}{ Treatment } & \multirow{2}{*}{$\begin{array}{c}\text { Packaging } \\
\text { Material }\end{array}$} & \multicolumn{5}{|c|}{$\begin{array}{c}\text { Storage } \\
\text { Period }\end{array}$} \\
\hline & & & $S_{1}$ & $\mathbf{S}_{2}$ & $\mathbf{S}_{3}$ & $\mathbf{S}_{4}$ & $\mathbf{S}_{5}$ \\
\hline \multirow{16}{*}{$\mathrm{V}_{1}$} & \multirow{4}{*}{$\mathrm{T}_{1}$} & $\mathrm{P}_{1}$ & $18.428 \pm 0.004$ & $18.429 \pm 0.004$ & $18.428 \pm 0.025$ & $18.428 \pm 0.0012$ & $18.429 \pm 0.047$ \\
\hline & & $\mathrm{P}_{2}$ & $18.428 \pm 0.004$ & $18.490 \pm 0.014$ & $18.551 \pm 0.024$ & $18.598 \pm 0.014$ & $18.652 \pm 0.025$ \\
\hline & & $\mathrm{P}_{3}$ & $18.428 \pm 0.004$ & $18.340 \pm 0.040$ & $19.030 \pm 0.015$ & $18.551 \pm 0.016$ & $18.652 \pm 0.034$ \\
\hline & & $\mathrm{P}_{4}$ & $18.428 \pm 0.004$ & $18.440 \pm 0.012$ & $18.490 \pm 0.034$ & $18.551 \pm 0.015$ & $18.500 \pm 0.054$ \\
\hline & \multirow{4}{*}{$\mathrm{T}_{2}$} & $\mathrm{P}_{1}$ & $19.030 \pm 0.003$ & $19.030 \pm 0.025$ & $19.030 \pm 0.016$ & $18.938 \pm 0.013$ & $18.999 \pm 0.024$ \\
\hline & & $\mathrm{P}_{2}$ & $19.030 \pm 0.003$ & $19.370 \pm 0.024$ & $19.500 \pm 0.027$ & $19.500 \pm 0.013$ & $19.530 \pm 0.031$ \\
\hline & & $\mathrm{P}_{3}$ & $19.030 \pm 0.003$ & $19.030 \pm 0.026$ & $20.000 \pm 0.024$ & $19.500 \pm 0.014$ & $19.530 \pm 0.015$ \\
\hline & & $\mathrm{P}_{4}$ & $19.030 \pm 0.003$ & $19.030 \pm 0.025$ & $19.040 \pm 0.025$ & $19.070 \pm 0.018$ & $19.090 \pm 0.001$ \\
\hline & \multirow{4}{*}{$\mathrm{T}_{3}$} & $\mathrm{P}_{1}$ & $20.000 \pm 0.010$ & $19.990 \pm 0.004$ & $20.010 \pm 0.029$ & $19.999 \pm 0.015$ & $20.010 \pm 0.005$ \\
\hline & & $\mathrm{P}_{2}$ & $20.000 \pm 0.010$ & $20.020 \pm 0.015$ & $20.120 \pm 0.034$ & $20.200 \pm 0.011$ & $20.120 \pm 0.004$ \\
\hline & & $\mathrm{P}_{3}$ & $20.000 \pm 0.010$ & $20.000 \pm 0.005$ & $20.000 \pm 0.015$ & $20.120 \pm 0.011$ & $20.200 \pm 0.012$ \\
\hline & & $\mathrm{P}_{4}$ & $20.000 \pm 0.010$ & $20.120 \pm 0.016$ & $20.000 \pm 0.019$ & $20.090 \pm 0.014$ & $20.400 \pm 0.034$ \\
\hline & \multirow{4}{*}{$\mathrm{T}_{4}$} & $\mathrm{P}_{1}$ & $20.010 \pm 0.024$ & $20.020 \pm 0.010$ & $20.000 \pm 0.017$ & $19.999 \pm 0.018$ & $20.010 \pm 0.025$ \\
\hline & & $\mathrm{P}_{2}$ & $20.010 \pm 0.024$ & $20.090 \pm 0.020$ & $20.000 \pm 0.023$ & $19.540 \pm 0.017$ & $19.870 \pm 0.210$ \\
\hline & & $\mathrm{P}_{3}$ & $20.010 \pm 0.024$ & $20.000 \pm 0.040$ & $18.428 \pm 0.034$ & $20.000 \pm 0015$ & $19.870 \pm 0.030$ \\
\hline & & $\mathrm{P}_{4}$ & $20.010 \pm 0.024$ & $20.020 \pm 0.010$ & $20.090 \pm 0.034$ & $20.010 \pm 0.002$ & $20.120 \pm 0.001$ \\
\hline \multirow{16}{*}{$\mathbf{V}_{2}$} & \multirow{4}{*}{$\mathrm{T}_{1}$} & $\mathrm{P}_{1}$ & $19.806 \pm 0.001$ & $19.807 \pm 0.005$ & $19.807 \pm 0.031$ & $19.806 \pm 0.003$ & $19.807 \pm 0.023$ \\
\hline & & $\mathrm{P}_{2}$ & $19.806 \pm 0.001$ & $19.800 \pm 0.004$ & $19.860 \pm 0.021$ & $19.731 \pm 0.012$ & $19.771 \pm 0.021$ \\
\hline & & $\mathrm{P}_{3}$ & $19.806 \pm 0.001$ & $19.670 \pm 0.004$ & $19.760 \pm 0.024$ & $19.860 \pm 0.002$ & $19.771 \pm 0.025$ \\
\hline & & $\mathrm{P}_{4}$ & $19.806 \pm 0.001$ & $19.820 \pm 0.004$ & $18.820 \pm 0.031$ & $19.860 \pm 0.001$ & $19.880 \pm 0.027$ \\
\hline & \multirow{4}{*}{$\mathrm{T}_{2}$} & $\mathrm{P}_{1}$ & $19.760 \pm 0.002$ & $19.770 \pm 0.001$ & $19.760 \pm 0.014$ & $19.770 \pm 0.004$ & $19.769 \pm 0.024$ \\
\hline & & $\mathrm{P}_{2}$ & $19.760 \pm 0.002$ & $18.990 \pm 0.003$ & $19.890 \pm 0.015$ & $19.960 \pm 0.012$ & $19.806 \pm 0.023$ \\
\hline & & $\mathrm{P}_{3}$ & $19.760 \pm 0.002$ & $19.760 \pm 0.003$ & $19.000 \pm 0.024$ & $19.890 \pm 0.002$ & $19.806 \pm 0.071$ \\
\hline & & $\mathrm{P}_{4}$ & $19.760 \pm 0.002$ & $19.760 \pm 0.001$ & $19.770 \pm 0.028$ & $17.790 \pm 0.014$ & $19.810 \pm 0.024$ \\
\hline & \multirow{4}{*}{$\mathrm{T}_{3}$} & $\mathrm{P}_{1}$ & $19.741 \pm 0.031$ & $19.742 \pm 0.001$ & $19.741 \pm 0.024$ & $19.742 \pm 0.025$ & $19.744 \pm 0.035$ \\
\hline & & $\mathrm{P}_{2}$ & $19.741 \pm 0.031$ & $19.747 \pm 0.005$ & $19.751 \pm 0.034$ & $19.755 \pm 0.031$ & $19.759 \pm 0.045$ \\
\hline & & $\mathrm{P}_{3}$ & $19.741 \pm 0.031$ & $19.741 \pm 0.008$ & $19.742 \pm 0.031$ & $19.741 \pm 0.025$ & $19.743 \pm 0.045$ \\
\hline & & $\mathrm{P}_{4}$ & $19.741 \pm 0.031$ & $19.740 \pm 0.014$ & $19.743 \pm 0.035$ & $19.743 \pm 0.034$ & $19.742 \pm 0.055$ \\
\hline & \multirow{4}{*}{$\mathrm{T}_{4}$} & $\mathrm{P}_{1}$ & $19.650 \pm 0.004$ & $19.660 \pm 0.018$ & $19.666 \pm 0.036$ & $19.659 \pm 0.014$ & $19.658 \pm 0.041$ \\
\hline & & $\mathrm{P}_{2}$ & $19.650 \pm 0.004$ & $19.760 \pm 0.08$ & $19.870 \pm 0.034$ & $20.052 \pm 0.025$ & $20.200 \pm 0.014$ \\
\hline & & $\mathrm{P}_{3}$ & $19.650 \pm 0.004$ & $19.650 \pm 0.004$ & $19.648 \pm 0.021$ & $19.843 \pm 0.036$ & $19.844 \pm 0.021$ \\
\hline & & $\mathrm{P}_{4}$ & $19.650 \pm 0.004$ & $19.651 \pm 0.014$ & $19.652 \pm 0.025$ & $19.652 \pm 0.037$ & $19.655 \pm 0.004$ \\
\hline \multirow{16}{*}{$\mathbf{V}_{3}$} & \multirow{4}{*}{$\mathrm{T}_{1}$} & $\mathrm{P}_{1}$ & $22.894 \pm 0.015$ & $22.899 \pm 0.005$ & $22.895 \pm 0.026$ & $22.894 \pm 0.035$ & $22.895 \pm 0.005$ \\
\hline & & $\mathrm{P}_{2}$ & $22.894 \pm 0.015$ & $22.880 \pm 0.017$ & $22.890 \pm 0.027$ & $22.894 \pm 0.021$ & $22.914 \pm 0.032$ \\
\hline & & $\mathrm{P}_{3}$ & $22.894 \pm 0.015$ & $22.896 \pm 0.027$ & $22.892 \pm 0.028$ & $22.890 \pm 0.025$ & $22.901 \pm 0.023$ \\
\hline & & $\mathrm{P}_{4}$ & $22.894 \pm 0.015$ & $22.880 \pm 0.015$ & $22.810 \pm 0.021$ & $22.840 \pm 0.024$ & $22.830 \pm 0.056$ \\
\hline & \multirow{4}{*}{$\mathrm{T}_{2}$} & $\mathrm{P}_{1}$ & $22.410 \pm 0.005$ & $22.419 \pm 0.006$ & $22.415 \pm 0.004$ & $22.410 \pm 0.039$ & $22.418 \pm 0.014$ \\
\hline & & $\mathrm{P}_{2}$ & $22.410 \pm 0.005$ & $22.430 \pm 0.014$ & $22.432 \pm 0.021$ & $22.439 \pm 0.048$ & $22.441 \pm 0.044$ \\
\hline & & $\mathrm{P}_{3}$ & $22.410 \pm 0.005$ & $22.410 \pm 0.015$ & $22.412 \pm 0.003$ & $22.414 \pm 0.048$ & $22.414 \pm 0.011$ \\
\hline & & $\mathrm{P}_{4}$ & $22.410 \pm 0.005$ & $22.410 \pm 0.014$ & $22.420 \pm 0.021$ & $22.410 \pm 0.014$ & $22.420 \pm 0.025$ \\
\hline & \multirow{4}{*}{$\mathrm{T}_{3}$} & $\mathrm{P}_{1}$ & $22.341 \pm 0.004$ & $22.344 \pm 0.012$ & $22.342 \pm 0.025$ & $22.341 \pm 0.047$ & $22.342 \pm 0.024$ \\
\hline & & $\mathrm{P}_{2}$ & $22.341 \pm 0.004$ & $22.346 \pm 0.012$ & $22.348 \pm 0.001$ & $22.351 \pm 0.024$ & $22.352 \pm 0.028$ \\
\hline & & $\mathrm{P}_{3}$ & $22.341 \pm 0.004$ & $22.341 \pm 0.025$ & $22.342 \pm 0.004$ & $22.341 \pm 0.028$ & $22.342 \pm 0.034$ \\
\hline & & $\mathrm{P}_{4}$ & $22.341 \pm 0.004$ & $22.341 \pm 0.026$ & $22.342 \pm 0.021$ & $22.344 \pm 0.025$ & $22.345 \pm 0.005$ \\
\hline & \multirow{4}{*}{$\mathrm{T}_{4}$} & $\mathrm{P}_{1}$ & $22.690 \pm 0.025$ & $22.710 \pm 0.025$ & $22.690 \pm 0.031$ & $22.701 \pm 0.026$ & $22.699 \pm 0.012$ \\
\hline & & $\mathrm{P}_{2}$ & $22.690 \pm 0.025$ & $22.710 \pm 0.013$ & $22.720 \pm 0.014$ & $22.730 \pm 0.025$ & $22.780 \pm 0.014$ \\
\hline & & $\mathrm{P}_{3}$ & $22.690 \pm 0.025$ & $22.690 \pm 0.014$ & $22.691 \pm 0.012$ & $22.693 \pm 0.004$ & $22.692 \pm 0.024$ \\
\hline & & $\mathrm{P}_{4}$ & $22.690 \pm 0.025$ & $22.692 \pm 0.012$ & $22.693 \pm 0.021$ & $22.691 \pm 0.024$ & $22.693 \pm 0.004$ \\
\hline
\end{tabular}

Effect of variety, treatments, packaging material and storage period on bulk density $(\mathrm{g} / \mathrm{ml})$ of brown rice

The changes in bulk density of brown rice due to the change in ambient temperature and relative humidity which affects the moisture content of brown rice and directly impact the bulk density of brown rice. PR-115 being bold and round had higher bulk density as compared to PR-118 and Punjab Mehak (Table 4.b). Hydrothermal treatments had a significant effect on bulk density of parboiled brown rice of all varieties. The individual effect of variety, treatment, storage period was found significant while packaging material had insignificant effect on bulk density of parboiled brown rice. The interaction of varieties with treatment, varieties with storage period, treatments with storage period and packaging materials with storage period had significant effect on bulk density of parboiled brown rice. Farhan (2011) reported that milling and parboiling had less significant effect on bulk density. The bulk density of raw rice increased while in parboiled milled rice slightly decreased. After parboiling a little decrease in bulk density of different varieties was observed. 
Table 4b Effect of variety, treatments, packaging material and storage period on bulk density $(\mathrm{g} / \mathrm{ml})$ of brown rice

\begin{tabular}{|c|c|c|c|c|c|c|c|}
\hline \multirow[t]{2}{*}{ Variety } & \multirow{2}{*}{ Treatment } & \multirow{2}{*}{$\begin{array}{c}\text { Packaging } \\
\text { Material }\end{array}$} & \multicolumn{5}{|c|}{$\begin{array}{c}\text { Storage } \\
\text { Period }\end{array}$} \\
\hline & & & $\mathbf{S}_{1}$ & $\mathbf{S}_{2}$ & $S_{3}$ & $\mathbf{S}_{4}$ & $\mathbf{S}_{5}$ \\
\hline \multirow{16}{*}{$\mathrm{V}_{1}$} & \multirow{4}{*}{$\mathrm{T}_{1}$} & $P_{1}$ & $0.782 \pm 0.002$ & $0.785 \pm 0.025$ & $0.788 \pm 0.020$ & $0.776 \pm 0.021$ & $0.771 \pm 0.003$ \\
\hline & & $\mathrm{P}_{2}$ & $0.782 \pm 0.002$ & $0.784 \pm 0.024$ & $0.787 \pm 0.021$ & $0.775 \pm 0.020$ & $0.771 \pm 0.002$ \\
\hline & & $\mathrm{P}_{3}$ & $0.782 \pm 0.002$ & $0.785 \pm 0.025$ & $0.787 \pm 0.021$ & $0.776 \pm 0.021$ & $0.770 \pm 0.023$ \\
\hline & & $\mathrm{P}_{4}$ & $0.782 \pm 0.002$ & $0.784 \pm 0.024$ & $0.788 \pm 0.020$ & $0.777 \pm 0.022$ & $0.770 \pm 0.023$ \\
\hline & \multirow{4}{*}{$\mathrm{T}_{2}$} & $P_{1}$ & $0.789 \pm 0.021$ & $0.792 \pm 0.015$ & $0.794 \pm 0.013$ & $0.788 \pm 0.025$ & $0.786 \pm 0.035$ \\
\hline & & $\mathrm{P}_{2}$ & $0.789 \pm 0.021$ & $0.791 \pm 0.014$ & $0.795 \pm 0.012$ & $0.788 \pm 0.026$ & $0.783 \pm 0.031$ \\
\hline & & $\mathrm{P}_{3}$ & $0.789 \pm 0.021$ & $0.792 \pm 0.015$ & $0.795 \pm 0.012$ & $0.786 \pm 0.025$ & $0.785 \pm 0.030$ \\
\hline & & $\mathrm{P}_{4}$ & $0.789 \pm 0.021$ & $0.793 \pm 0.012$ & $0.795 \pm 0.013$ & $0.784 \pm 0.031$ & $0.786 \pm 0.035$ \\
\hline & \multirow{4}{*}{$\mathrm{T}_{3}$} & $P_{1}$ & $0.881 \pm 0.003$ & $0.884 \pm 0.024$ & $0.794 \pm 0.013$ & $0.882 \pm 0.035$ & $0.882 \pm 0.036$ \\
\hline & & $\mathrm{P}_{2}$ & $0.881 \pm 0.003$ & $0.881 \pm 0.021$ & $0.790 \pm 0.021$ & $0.881 \pm 0.045$ & $0.880 \pm 0.033$ \\
\hline & & $\mathrm{P}_{3}$ & $0.881 \pm 0.003$ & $0.880 \pm 0.021$ & $0.791 \pm 0.023$ & $0.882 \pm 0.035$ & $0.881 \pm 0.032$ \\
\hline & & $\mathrm{P}_{4}$ & $0.881 \pm 0.003$ & $0.882 \pm 0.004$ & $0.793 \pm 0.022$ & $0.882 \pm 0.034$ & $0.880 \pm 0.021$ \\
\hline & \multirow{4}{*}{$\mathrm{T}_{4}$} & $\mathrm{P}_{1}$ & $0.897 \pm 0.025$ & $0.899 \pm 0.025$ & $0.895 \pm 0.024$ & $0.893 \pm 0.001$ & $0.890 \pm 0.012$ \\
\hline & & $\mathrm{P}_{2}$ & $0.897 \pm 0.025$ & $0.898 \pm 0.021$ & $0.898 \pm 0.022$ & $0.893 \pm 0.001$ & $0.890 \pm 0.011$ \\
\hline & & $\mathrm{P}_{3}$ & $0.897 \pm 0.025$ & $0.896 \pm 0.022$ & $0.896 \pm 0.023$ & $0.895 \pm 0.025$ & $0.892 \pm 0.002$ \\
\hline & & $\mathrm{P}_{4}$ & $0.897 \pm 0.025$ & $0.897 \pm 0.024$ & $0.896 \pm 0.022$ & $0.893 \pm 0.001$ & $0.893 \pm 0.001$ \\
\hline \multirow{16}{*}{$\mathbf{V}_{2}$} & \multirow{4}{*}{$\mathrm{T}_{1}$} & $\mathrm{P}_{1}$ & $0.785 \pm 0.003$ & $0.785 \pm 0.013$ & $0.787 \pm 0.021$ & $0.787 \pm 0.035$ & $0.784 \pm 0.025$ \\
\hline & & $\mathrm{P}_{2}$ & $0.785 \pm 0.003$ & $0.786 \pm 0.012$ & $0.788 \pm 0.020$ & $0.785 \pm 0.001$ & $0.784 \pm 0.031$ \\
\hline & & $\mathrm{P}_{3}$ & $0.785 \pm 0.003$ & $0.785 \pm 0.013$ & $0.788 \pm 0.020$ & $0.788 \pm 0.021$ & $0.783 \pm 0.001$ \\
\hline & & $\mathrm{P}_{4}$ & $0.785 \pm 0.003$ & $0.786 \pm 0.012$ & $0.785 \pm 0.003$ & $0.780 \pm 0.023$ & $0.780 \pm 0.024$ \\
\hline & \multirow{4}{*}{$\mathrm{T}_{2}$} & $\mathrm{P}_{1}$ & $0.784 \pm 0.001$ & $0.782 \pm 0.002$ & $0.785 \pm 0.003$ & $0.787 \pm 0.024$ & $0.784 \pm 0.012$ \\
\hline & & $\mathrm{P}_{2}$ & $0.784 \pm 0.001$ & $0.785 \pm 0.003$ & $0.788 \pm 0.001$ & $0.776 \pm 0.021$ & $0.771 \pm 0.003$ \\
\hline & & $\mathrm{P}_{3}$ & $0.784 \pm 0.001$ & $0.782 \pm 0.002$ & $0.784 \pm 0.024$ & $0.788 \pm 0.023$ & $0.782 \pm 0.001$ \\
\hline & & $\mathrm{P}_{4}$ & $0.784 \pm 0.001$ & $0.788 \pm 0.001$ & $0.776 \pm 0.026$ & $0.771 \pm 0.025$ & $0.770 \pm 0.002$ \\
\hline & \multirow{4}{*}{$\mathrm{T}_{3}$} & $\mathrm{P}_{1}$ & $0.834 \pm 0.002$ & $0.835 \pm 0.013$ & $0.837 \pm 0.032$ & $0.831 \pm 0.003$ & $0.830 \pm 0.051$ \\
\hline & & $\mathrm{P}_{2}$ & $0.834 \pm 0.002$ & $0.839 \pm 0.015$ & $0.841 \pm 0.022$ & $0.840 \pm 0.024$ & $0.836 \pm 0.023$ \\
\hline & & $\mathrm{P}_{3}$ & $0.834 \pm 0.002$ & $0.834 \pm 0.012$ & $0.838 \pm 0.014$ & $0.841 \pm 0.002$ & $0.837 \pm 0.024$ \\
\hline & & $\mathrm{P}_{4}$ & $0.834 \pm 0.002$ & $0.836 \pm 0.016$ & $0.843 \pm 0.025$ & $0.844 \pm 0.001$ & $0.840 \pm 0.014$ \\
\hline & \multirow{4}{*}{$\mathrm{T}_{4}$} & $\mathrm{P}_{1}$ & $0.819 \pm 0.031$ & $0.819 \pm 0.025$ & $0.821 \pm 0.035$ & $0.823 \pm 0.002$ & $0.825 \pm 0.015$ \\
\hline & & $\mathrm{P}_{2}$ & $0.819 \pm 0.031$ & $0.820 \pm 0.024$ & $0.822 \pm 0.025$ & $0.818 \pm 0.045$ & $0.818 \pm 0.025$ \\
\hline & & $\mathrm{P}_{3}$ & $0.819 \pm 0.031$ & $0.822 \pm 0.025$ & $0.824 \pm 0.031$ & $0.816 \pm 0.002$ & $0.819 \pm 0.021$ \\
\hline & & $\mathrm{P}_{4}$ & $0.819 \pm 0.031$ & $0.821 \pm 0.023$ & $0.823 \pm 0.030$ & $0.820 \pm 0.035$ & $0.820 \pm 0.034$ \\
\hline \multirow{16}{*}{$\mathbf{V}_{3}$} & \multirow{4}{*}{$\mathrm{T}_{1}$} & $P_{1}$ & $0.786 \pm 0.002$ & $0.786 \pm 0.002$ & $0.788 \pm 0.001$ & $0.785 \pm 0.001$ & $0.784 \pm 0.002$ \\
\hline & & $\mathrm{P}_{2}$ & $0.786 \pm 0.002$ & $0.787 \pm 0.021$ & $0.787 \pm 0.002$ & $0.784 \pm 0.002$ & $0.784 \pm 0.001$ \\
\hline & & $\mathrm{P}_{3}$ & $0.786 \pm 0.002$ & $0.786 \pm 0.002$ & $0.786 \pm 0.014$ & $0.785 \pm 0.001$ & $0.780 \pm 0.037$ \\
\hline & & $\mathrm{P}_{4}$ & $0.786 \pm 0.002$ & $0.788 \pm 0.013$ & $0.788 \pm 0.015$ & $0.789 \pm 0.021$ & $0.783 \pm 0.031$ \\
\hline & \multirow{4}{*}{$\mathrm{T}_{2}$} & $P_{1}$ & $0.798 \pm 0.012$ & $0.798 \pm 0.024$ & $0.801 \pm 0.002$ & $0.805 \pm 0.001$ & $0.803 \pm 0.002$ \\
\hline & & $\mathrm{P}_{2}$ & $0.798 \pm 0.012$ & $0.799 \pm 0.025$ & $0.802 \pm 0.002$ & $0.805 \pm 0.001$ & $0.802 \pm 0.001$ \\
\hline & & $\mathrm{P}_{3}$ & $0.798 \pm 0.012$ & $0.798 \pm 0.021$ & $0.802 \pm 0.002$ & $0.802 \pm 0.002$ & $0.802 \pm 0.002$ \\
\hline & & $\mathrm{P}_{4}$ & $0.798 \pm 0.012$ & $0.797 \pm 0.022$ & $0.799 \pm 0.034$ & $0.804 \pm 0.003$ & $0.801 \pm 0.002$ \\
\hline & \multirow{4}{*}{$\mathrm{T}_{3}$} & $\mathrm{P}_{1}$ & $0.801 \pm 0.002$ & $0.802 \pm 0.014$ & $0.804 \pm 0.031$ & $0.804 \pm 0.024$ & $0.802 \pm 0.002$ \\
\hline & & $\mathrm{P}_{2}$ & $0.801 \pm 0.002$ & $0.807 \pm 0.012$ & $0.809 \pm 0.032$ & $0.807 \pm 0.025$ & $0.805 \pm 0.001$ \\
\hline & & $\mathrm{P}_{3}$ & $0.801 \pm 0.002$ & $0.802 \pm 0.014$ & $0.803 \pm 0.025$ & $0.802 \pm 0.003$ & $0.801 \pm 0.002$ \\
\hline & & $\mathrm{P}_{4}$ & $0.801 \pm 0.002$ & $0.803 \pm 0.011$ & $0.804 \pm 0.029$ & $0.804 \pm 0.001$ & $0.803 \pm 0.001$ \\
\hline & \multirow{4}{*}{$\mathrm{T}_{4}$} & $\mathrm{P}_{1}$ & $0.853 \pm 0.001$ & $0.855 \pm 0.027$ & $0.857 \pm 0.041$ & $0.858 \pm 0.047$ & $0.852 \pm 0.002$ \\
\hline & & $\mathrm{P}_{2}$ & $0.853 \pm 0.001$ & $0.854 \pm 0.028$ & $0.855 \pm 0.001$ & $0.853 \pm 0.011$ & $0.851 \pm 0.001$ \\
\hline & & $\mathrm{P}_{3}$ & $0.853 \pm 0.001$ & $0.853 \pm 0.024$ & $0.853 \pm 0.001$ & $0.852 \pm 0.013$ & $0.851 \pm 0.002$ \\
\hline & & $\mathrm{P}_{4}$ & $0.853 \pm 0.001$ & $0.855 \pm 0.027$ & $0.855 \pm 0.001$ & $0.857 \pm 0.012$ & $0.853 \pm 0.001$ \\
\hline
\end{tabular}

$\mathrm{CD}(\mathrm{p} \leq 0.05): \mathrm{V}: 0.32, \mathrm{~T}: 0.16, \mathrm{~S}: 0.09, \mathrm{P}: \mathrm{NS}, \mathrm{V} \times \mathrm{T}: 0.22, \mathrm{~V} \times \mathrm{S}: 0.29, \mathrm{~T} \times \mathrm{S}: 0.29, \mathrm{P} \times \mathrm{S}: 0.33$

Effect of variety, treatments, packaging material and storage period on color profile analysis ( $\mathrm{L}$, a and $\mathrm{b}$ values) of parboiled brown rice affect the L-value significantly. L-value decreased from 57.90 to 54.11 during storage in case of $\mathrm{T}_{1}$ of variety PR-115.

Color differences in hydrothermally treated parboiled brown rice were instrumentally measured by Hunter Lab Calorimeter. L, a, b values for brown rice were obtained in triplicate. ' $L$ ' depicts lightness and its value varies from 0 to 100 ; ' $+\mathrm{a}$ ' depicts redness and '-a' depicts greenness; ' $+\mathrm{b}$ ' depicts yellowness and ' $-b$ ' depicts blueness. Significant difference in the color analysis in case of ' $L$ ' value was observed (Table 5.a). L-value decreased with hydrothermal treatments which depicted that these treatments the color of brown rice becomes lighter. L-value decreased with storage however packaging material did not

Table 5a Effect of variety, treatments, packaging material and storage period on color (1) of brown rice 


\begin{tabular}{|c|c|c|c|c|c|c|c|}
\hline \multirow[t]{2}{*}{ Variety } & \multirow[t]{2}{*}{ Treatment } & \multirow{2}{*}{$\begin{array}{l}\text { Packaging } \\
\text { material }\end{array}$} & \multicolumn{5}{|c|}{$\begin{array}{l}\text { Storage } \\
\text { Period }\end{array}$} \\
\hline & & & $S_{1}$ & $\mathbf{S}_{2}$ & $\mathbf{S}_{3}$ & $\mathbf{S}_{4}$ & $\mathbf{S}_{5}$ \\
\hline \multirow{16}{*}{$\mathbf{V}_{1}$} & \multirow{4}{*}{$\mathrm{T}_{1}$} & $\mathrm{P}_{1}$ & $57.90 \pm 3.15$ & $57.24 \pm 1.26$ & $55.73 \pm 1.36$ & $54.71 \pm 1.25$ & $54.12 \pm 0.54$ \\
\hline & & $\mathrm{P}_{2}$ & $57.90 \pm 3.15$ & $57.26 \pm 1.11$ & $55.78 \pm 1.25$ & $54.78 \pm 1.36$ & $54.13 \pm 1.54$ \\
\hline & & $\mathrm{P}_{3}$ & $57.90 \pm 3.15$ & $57.26 \pm 2.15$ & $55.74 \pm 1.89$ & $54.73 \pm 1.85$ & $54.12 \pm 1.46$ \\
\hline & & $\mathrm{P}_{4}$ & $57.90 \pm 3.15$ & $57.25 \pm 2.35$ & $55.79 \pm 1.36$ & $54.70 \pm 1.45$ & $54.11 \pm 1.96$ \\
\hline & \multirow{4}{*}{$\mathrm{T}_{2}$} & $P_{1}$ & $53.80 \pm 3.06$ & $53.10 \pm 2.14$ & $52.40 \pm 0.25$ & $51.50 \pm 1.64$ & $51.20 \pm 1.56$ \\
\hline & & $\mathrm{P}_{2}$ & $53.80 \pm 3.06$ & $53.10 \pm 2.09$ & $52.42 \pm 1.23$ & $51.55 \pm 1.56$ & $51.20 \pm 1.45$ \\
\hline & & $\mathrm{P}_{3}$ & $53.80 \pm 3.06$ & $53.20 \pm 2.15$ & $55.25 \pm 1.65$ & $51.56 \pm 1.54$ & $51.22 \pm 0.89$ \\
\hline & & $\mathrm{P}_{4}$ & $53.80 \pm 3.06$ & $53.10 \pm 2.07$ & $55.26 \pm 1.75$ & $51.55 \pm 1.56$ & $51.23 \pm 1.45$ \\
\hline & \multirow{4}{*}{$\mathrm{T}_{3}$} & $\mathrm{P}_{1}$ & $52.67 \pm 2.59$ & $51.34 \pm 1.59$ & $50.15 \pm 1.89$ & $49.84 \pm 1.45$ & $49.15 \pm 0.65$ \\
\hline & & $\mathrm{P}_{2}$ & $52.67 \pm 2.59$ & $51.33 \pm 1.48$ & $50.16 \pm 1.45$ & $49.85 \pm 1.98$ & $49.16 \pm 1.45$ \\
\hline & & $\mathrm{P}_{3}$ & $52.67 \pm 2.59$ & $51.34 \pm 1.12$ & $50.14 \pm 1.89$ & $49.85 \pm 1.56$ & $49.15 \pm 0.56$ \\
\hline & & $\mathrm{P}_{4}$ & $52.67 \pm 2.59$ & $51.35 \pm 1.35$ & $50.16 \pm 1.56$ & $49.85 \pm 1.24$ & $49.15 \pm 1.45$ \\
\hline & \multirow{4}{*}{$\mathrm{T}_{4}$} & $P_{1}$ & $47.19 \pm 1.48$ & $47.01 \pm 1.41$ & $46.82 \pm 1.36$ & $46.17 \pm 1.14$ & $45.99 \pm 1.57$ \\
\hline & & $\mathrm{P}_{2}$ & $47.19 \pm 1.48$ & $47.01 \pm 1.39$ & $46.84 \pm 1.26$ & $46.18 \pm 1.26$ & $45.98 \pm 1.36$ \\
\hline & & $\mathrm{P}_{3}$ & $47.19 \pm 1.48$ & $47.02 \pm 1.78$ & $46.85 \pm 1.65$ & $46.18 \pm 1.25$ & $45.95 \pm 1.69$ \\
\hline & & $\mathrm{P}_{4}$ & $47.19 \pm 1.48$ & $47.03 \pm 2.04$ & $46.82 \pm 1.54$ & $46.19 \pm 1.36$ & $45.96 \pm 1.58$ \\
\hline \multirow{16}{*}{$\mathbf{V}_{2}$} & \multirow{4}{*}{$\mathrm{T}_{1}$} & $\mathrm{P}_{1}$ & $57.76 \pm 3.24$ & $57.34 \pm 2.16$ & $55.85 \pm 1.78$ & $55.18 \pm 1.24$ & $54.87 \pm 1.85$ \\
\hline & & $\mathrm{P}_{2}$ & $57.76 \pm 3.24$ & $57.34 \pm 2.57$ & $55.85 \pm 1.56$ & $55.19 \pm 1.25$ & $54.85 \pm 1.45$ \\
\hline & & $\mathrm{P}_{3}$ & $57.76 \pm 3.24$ & $57.35 \pm 2.03$ & $55.86 \pm 1.36$ & $55.18 \pm 1.45$ & $54.84 \pm 1.36$ \\
\hline & & $\mathrm{P}_{4}$ & $57.76 \pm 3.24$ & $57.35 \pm 1.54$ & $55.85 \pm 0.25$ & $55.17 \pm 1.45$ & $54.84 \pm 1.56$ \\
\hline & \multirow{4}{*}{$\mathrm{T}_{2}$} & $\mathrm{P}_{1}$ & $56.89 \pm 3.04$ & $55.90 \pm 1.59$ & $54.19 \pm 1.45$ & $53.87 \pm 1.36$ & $53.12 \pm 1.13$ \\
\hline & & $\mathrm{P}_{2}$ & $56.89 \pm 3.04$ & $55.80 \pm 1.03$ & $54.19 \pm 1.25$ & $53.86 \pm 1.56$ & $53.13 \pm 1.12$ \\
\hline & & $\mathrm{P}_{3}$ & $56.89 \pm 3.04$ & $55.80 \pm 1.25$ & $55.18 \pm 1.85$ & $53.85 \pm 1.25$ & $53.15 \pm 1.36$ \\
\hline & & $\mathrm{P}_{4}$ & $56.89 \pm 3.04$ & $55.90 \pm 1.31$ & $55.16 \pm 1.45$ & $53.85 \pm 1.45$ & $53.15 \pm 1.65$ \\
\hline & \multirow{4}{*}{$\mathrm{T}_{3}$} & $\mathrm{P}_{1}$ & $56.78 \pm 2.59$ & $56.41 \pm 2.14$ & $55.63 \pm 1.35$ & $54.05 \pm 0.65$ & $53.81 \pm 1.45$ \\
\hline & & $\mathrm{P}_{2}$ & $56.78 \pm 2.59$ & $56.42 \pm 2.01$ & $55.64 \pm 1.64$ & $54.04 \pm 1.25$ & $53.82 \pm 1.36$ \\
\hline & & $\mathrm{P}_{3}$ & $56.78 \pm 2.59$ & $56.42 \pm 2.03$ & $55.64 \pm 0.46$ & $55.01 \pm 1.45$ & $53.81 \pm 1.25$ \\
\hline & & $\mathrm{P}_{4}$ & $56.78 \pm 2.59$ & $56.41 \pm 1.29$ & $55.65 \pm 1.34$ & $55.01 \pm 1.36$ & $53.83 \pm 1.63$ \\
\hline & \multirow{4}{*}{$\mathrm{T}_{4}$} & $P_{1}$ & $49.77 \pm 1.41$ & $49.41 \pm 1.48$ & $48.58 \pm 1.25$ & $47.16 \pm 1.25$ & $47.02 \pm 1.45$ \\
\hline & & $\mathrm{P}_{2}$ & $49.77 \pm 1.41$ & $49.42 \pm 1.44$ & $48.56 \pm 1.56$ & $47.19 \pm 1.14$ & $47.05 \pm 1.25$ \\
\hline & & $\mathrm{P}_{3}$ & $49.77 \pm 1.41$ & $49.41 \pm 1.36$ & $48.56 \pm 1.36$ & $47.15 \pm 1.56$ & $47.02 \pm 1.89$ \\
\hline & & $\mathrm{P}_{4}$ & $49.77 \pm 1.41$ & $49.43 \pm 1.29$ & $48.59 \pm 1.45$ & $47.18 \pm 1.56$ & $47.05 \pm 1.45$ \\
\hline \multirow{16}{*}{$\mathbf{V}_{3}$} & \multirow{4}{*}{$\mathrm{T}_{1}$} & $\mathrm{P}_{1}$ & $57.02 \pm 2.54$ & $56.87 \pm 1.32$ & $56.21 \pm 0.25$ & $55.55 \pm 1.24$ & $55.21 \pm 1.25$ \\
\hline & & $\mathrm{P}_{2}$ & $57.02 \pm 2.54$ & $56.85 \pm 1.06$ & $56.21 \pm 1.89$ & $55.54 \pm 51.05$ & $55.22 \pm 1.45$ \\
\hline & & $\mathrm{P}_{3}$ & $57.02 \pm 2.54$ & $56.85 \pm 1.49$ & $56.23 \pm 1.25$ & $55.55 \pm 1.89$ & $55.23 \pm 1.36$ \\
\hline & & $\mathrm{P}_{4}$ & $57.02 \pm 2.54$ & $56.84 \pm 1.22$ & $56.22 \pm 0.55$ & $55.55 \pm 1.25$ & $55.24 \pm 1.25$ \\
\hline & \multirow{4}{*}{$\mathrm{T}_{2}$} & $P_{1}$ & $55.42 \pm 3.14$ & $55.01 \pm 1.09$ & $54.64 \pm 1.25$ & $53.78 \pm 1.36$ & $53.37 \pm 0.36$ \\
\hline & & $\mathrm{P}_{2}$ & $55.42 \pm 3.14$ & $55.00 \pm 0.98$ & $54.65 \pm 1.54$ & $53.75 \pm 1.35$ & $53.35 \pm 1.54$ \\
\hline & & $\mathrm{P}_{3}$ & $55.42 \pm 3.14$ & $55.01 \pm 1.26$ & $54.68 \pm 1.06$ & $53.76 \pm 1.25$ & $53.35 \pm 1.45$ \\
\hline & & $\mathrm{P}_{4}$ & $55.42 \pm 3.14$ & $55.02 \pm 1.47$ & $54.65 \pm 1.02$ & $53.76 \pm 1.36$ & $53.36 \pm 1.25$ \\
\hline & \multirow{4}{*}{$\mathrm{T}_{3}$} & $\mathrm{P}_{1}$ & $53.26 \pm 3.29$ & $52.18 \pm 1.36$ & $51.69 \pm 1.65$ & $51.21 \pm 1.45$ & $50.12 \pm 1.12$ \\
\hline & & $\mathrm{P}_{2}$ & $53.26 \pm 3.29$ & $52.15 \pm 1.25$ & $51.69 \pm 1.25$ & $51.22 \pm 1.25$ & $50.16 \pm 1.65$ \\
\hline & & $\mathrm{P}_{3}$ & $53.26 \pm 3.29$ & $52.16 \pm 1.54$ & $51.68 \pm 1.54$ & $51.23 \pm 0.25$ & $50.12 \pm 1.25$ \\
\hline & & $\mathrm{P}_{4}$ & $53.26 \pm 3.29$ & $52.17 \pm 1.25$ & $51.68 \pm 1.65$ & $51.22 \pm 1.36$ & $50.13 \pm 1.45$ \\
\hline & \multirow{4}{*}{$\mathrm{T}_{4}$} & $\mathrm{P}_{1}$ & $52.12 \pm 1.55$ & $51.86 \pm 1.85$ & $51.13 \pm 1.25$ & $50.74 \pm 1.25$ & $50.44 \pm 1.25$ \\
\hline & & $\mathrm{P}_{2}$ & $52.12 \pm 1.55$ & $51.85 \pm 1.02$ & $51.16 \pm 1.35$ & $50.75 \pm 1.36$ & $50.45 \pm 1.56$ \\
\hline & & $\mathrm{P}_{3}$ & $52.12 \pm 1.55$ & $51.85 \pm 1.30$ & $51.15 \pm 1.54$ & $50.76 \pm 1.45$ & $50.46 \pm 1.36$ \\
\hline & & $\mathrm{P}_{4}$ & $52.12 \pm 1.55$ & $51.85 \pm 1.58$ & $51.15 \pm 1.25$ & $50.74 \pm 1.89$ & $50.44 \pm 1.26$ \\
\hline
\end{tabular}

Among the varieties PR-118 had minimum L-value for $\mathrm{T}_{4}$ treatment as compared to other treatments of PR-115 and PR-118 varieties after parboiling. The individual effect of variety, treatment, storage period was found significant while packaging material insignificant effect on L-value of parboiled brown rice. The interactions of varieties with treatment, varieties with storage period, treatments with storage period and packaging materials with storage period were varied significantly while varied insignificantly with respect to L-value of parboiled brown rice. The $\mathrm{L}$-value of parboiled brown rice decreased with increased storage periods.

Kimura et al (1993) reported that whiteness of parboiled rice significantly affected the temperature and period of soaking and steaming influence
(Parnsakhom and Noomhorm, 2008). These results revealed that lower steaming temperature and time were favorable to produce a better quality of parboiled brown rice (Islam et al. 2002).

Data for ' $a$ *' value of color of parboiled brown rice were presented in Table 5.b. Hydrothermal treatment increased the ' $a *$ ' value to quiet large extent from 2.2 in case of control to 2.56 in $\mathrm{T}_{4}$ variety $\mathrm{V}_{1}$ however the effect of storage on $\mathrm{a}^{*}$ ' value was opposite. The ' $\mathrm{a}^{*}$ ' value decreased upon storage which means redness of sample decreased with storage. The packaging material did not have certain effect on ' $a$ *' value of color of parboiled brown rice. Among the varieties Punjab Mehak had maximum ' $a$ *' value for $T_{4}$ treatment brown rice after the hydrothermal treatment as compared to PR-115 and PR-118. The individual 
effect of variety, treatment, storage period was significant while individual effect of packaging material was insignificant on ' $a$ *' value of parboiled brown rice. The interaction of varieties with treatment, varieties with storage period and treatments with storage period had significant effect while interaction of packaging materials with storage period had insignificant effect on ' $a$ *' value of parboiled brown rice. The ' $a$ *' value of parboiled brown rice decreased with increased storage periods.

Table $\mathbf{5 b}$ Effect of variety, treatments, packaging material and storage period on color $\left(\mathrm{a}^{*}\right)$ of brown rice

\begin{tabular}{|c|c|c|c|c|c|c|c|}
\hline \multirow{2}{*}{ Variety } & \multirow{2}{*}{ Treatment } & \multirow{2}{*}{$\begin{array}{l}\text { Packaging } \\
\text { Material }\end{array}$} & \multicolumn{5}{|c|}{ Storage Period } \\
\hline & & & $S_{1}$ & $\mathbf{S}_{2}$ & $\mathbf{S}_{3}$ & $\mathbf{S}_{4}$ & $\mathbf{S}_{5}$ \\
\hline \multirow{16}{*}{$\mathrm{V}_{1}$} & \multirow{4}{*}{$\mathrm{T}_{1}$} & $\mathrm{P}_{1}$ & $2.20 \pm 0.01$ & $2.13 \pm 0.25$ & $1.44 \pm 0.01$ & $1.22 \pm 0.11$ & $1.12 \pm 0.11$ \\
\hline & & $\mathrm{P}_{2}$ & $2.20 \pm 0.01$ & $2.13 \pm 0.36$ & $1.45 \pm 0.06$ & $1.21 \pm 0.01$ & $1.13 \pm 0.02$ \\
\hline & & $\mathrm{P}_{3}$ & $2.20 \pm 0.01$ & $2.15 \pm 0.45$ & $1.44 \pm 0.02$ & $1.22 \pm 0.03$ & $1.13 \pm 0.03$ \\
\hline & & $\mathrm{P}_{4}$ & $2.20 \pm 0.01$ & $2.15 \pm 0.45$ & $1.44 \pm 0.03$ & $1.21 \pm 0.01$ & $1.12 \pm 0.03$ \\
\hline & \multirow{4}{*}{$\mathrm{T}_{2}$} & $\mathrm{P}_{1}$ & $2.30 \pm 0.02$ & $2.06 \pm 0.26$ & $1.59 \pm 0.01$ & $1.20 \pm 0.02$ & $1.01 \pm 0.03$ \\
\hline & & $\mathrm{P}_{2}$ & $2.30 \pm 0.02$ & $2.05 \pm 0.13$ & $1.61 \pm 0.11$ & $1.23 \pm 0.04$ & $1.02 \pm 0.02$ \\
\hline & & $\mathrm{P}_{3}$ & $2.30 \pm 0.02$ & $2.04 \pm 0.45$ & $1.60 \pm 0.02$ & $1.22 \pm 0.05$ & $1.01 \pm 0.01$ \\
\hline & & $\mathrm{P}_{4}$ & $2.30 \pm 0.02$ & $2.01 \pm 0.14$ & $1.60 \pm 0.03$ & $1.23 \pm 0.04$ & $1.01 \pm 0.02$ \\
\hline & \multirow{4}{*}{$\mathrm{T}_{3}$} & $\mathrm{P}_{1}$ & $2.43 \pm 0.11$ & $2.22 \pm 0.04$ & $1.87 \pm 0.11$ & $1.69 \pm 0.03$ & $1.33 \pm 0.05$ \\
\hline & & $\mathrm{P}_{2}$ & $2.43 \pm 0.11$ & $2.22 \pm 0.14$ & $1.85 \pm 0.04$ & $1.68 \pm 0.02$ & $1.32 \pm 0.06$ \\
\hline & & $\mathrm{P}_{3}$ & $2.43 \pm 0.11$ & $2.25 \pm 0.17$ & $1.87 \pm 0.05$ & $1.67 \pm 0.08$ & $1.33 \pm 0.05$ \\
\hline & & $\mathrm{P}_{4}$ & $2.43 \pm 0.11$ & $2.24 \pm 0.05$ & $1.86 \pm 0.16$ & $1.68 \pm 0.06$ & $1.31 \pm 0.02$ \\
\hline & \multirow{4}{*}{$\mathrm{T}_{4}$} & $\mathrm{P}_{1}$ & $2.56 \pm 0.03$ & $1.79 \pm 0.05$ & $1.90 \pm 0.15$ & $1.79 \pm 0.04$ & $1.38 \pm 0.04$ \\
\hline & & $\mathrm{P}_{2}$ & $2.56 \pm 0.03$ & $1.78 \pm 0.47$ & $1.91 \pm 0.14$ & $1.77 \pm 0.03$ & $1.37 \pm 0.02$ \\
\hline & & $\mathrm{P}_{3}$ & $2.56 \pm 0.03$ & $1.79 \pm 0.06$ & $1.92 \pm 0.24$ & $1.78 \pm 0.04$ & $1.35 \pm 0.02$ \\
\hline & & $\mathrm{P}_{4}$ & $2.56 \pm 0.03$ & $1.79 \pm 0.14$ & $1.93 \pm 0.01$ & $1.79 \pm 0.12$ & $1.38 \pm 0.03$ \\
\hline \multirow{16}{*}{$\mathbf{V}_{2}$} & \multirow{4}{*}{$\mathrm{T}_{1}$} & $\mathrm{P}_{1}$ & $2.04 \pm 0.12$ & $1.81 \pm 0.09$ & $1.66 \pm 0.02$ & $1.32 \pm 0.11$ & $1.09 \pm 0.01$ \\
\hline & & $\mathrm{P}_{2}$ & $2.04 \pm 0.12$ & $1.80 \pm 0.25$ & $1.65 \pm 0.02$ & $1.31 \pm 0.02$ & $1.08 \pm 0.01$ \\
\hline & & $\mathrm{P}_{3}$ & $2.04 \pm 0.12$ & $1.80 \pm 0.89$ & $1.65 \pm 0.01$ & $1.31 \pm 0.02$ & $1.07 \pm 0.01$ \\
\hline & & $\mathrm{P}_{4}$ & $2.04 \pm 0.12$ & $1.81 \pm 0.36$ & $1.67 \pm 0.03$ & $1.32 \pm 0.01$ & $1.08 \pm 0.02$ \\
\hline & \multirow{4}{*}{$\mathrm{T}_{2}$} & $\mathrm{P}_{1}$ & $2.11 \pm 0.06$ & $1.83 \pm 0.21$ & $1.25 \pm 0.06$ & $1.02 \pm 0.02$ & $0.88 \pm 0.02$ \\
\hline & & $\mathrm{P}_{2}$ & $2.11 \pm 0.06$ & $1.83 \pm 0.26$ & $1.23 \pm 0.05$ & $1.03 \pm 0.03$ & $0.87 \pm 0.03$ \\
\hline & & $\mathrm{P}_{3}$ & $2.11 \pm 0.06$ & $1.82 \pm 0.25$ & $1.23 \pm 0.01$ & $1.03 \pm 0.02$ & $0.88 \pm 0.03$ \\
\hline & & $\mathrm{P}_{4}$ & $2.11 \pm 0.06$ & $1.83 \pm 0.11$ & $1.24 \pm 0.02$ & $1.04 \pm 0.01$ & $0.88 \pm 0.03$ \\
\hline & \multirow{4}{*}{$\mathrm{T}_{3}$} & $\mathrm{P}_{1}$ & $2.15 \pm 0.01$ & $1.89 \pm 0.18$ & $1.77 \pm 0.01$ & $1.45 \pm 0.02$ & $1.15 \pm 0.02$ \\
\hline & & $\mathrm{P}_{2}$ & $2.15 \pm 0.01$ & $1.88 \pm 0.19$ & $1.75 \pm 0.01$ & $1.44 \pm 0.01$ & $1.14 \pm 0.11$ \\
\hline & & $\mathrm{P}_{3}$ & $2.15 \pm 0.01$ & $1.89 \pm 0.17$ & $1.77 \pm 0.11$ & $1.43 \pm 0.02$ & $1.14 \pm 0.02$ \\
\hline & & $\mathrm{P}_{4}$ & $2.15 \pm 0.01$ & $1.88 \pm 1.15$ & $1.76 \pm 0.02$ & $1.44 \pm 0.01$ & $1.12 \pm 0.02$ \\
\hline & \multirow{4}{*}{$\mathrm{T}_{4}$} & $\mathrm{P}_{1}$ & $2.53 \pm 0.04$ & $1.99 \pm 0.24$ & $1.83 \pm 0.12$ & $1.65 \pm 0.02$ & $1.29 \pm 0.01$ \\
\hline & & $\mathrm{P}_{2}$ & $2.53 \pm 0.04$ & $1.98 \pm 0.35$ & $1.83 \pm 0.11$ & $1.65 \pm 0.01$ & $1.28 \pm 0.11$ \\
\hline & & $\mathrm{P}_{3}$ & $2.53 \pm 0.04$ & $1.99 \pm 0.61$ & $1.83 \pm 0.02$ & I. $66 \pm 0.03$ & $1.29 \pm 0.21$ \\
\hline & & $\mathrm{P}_{4}$ & $2.53 \pm 0.04$ & $1.97 \pm 0.32$ & $1.82 \pm 0.01$ & $1.65 \pm 0.02$ & $1.26 \pm 0.02$ \\
\hline \multirow{16}{*}{$\mathbf{V}_{3}$} & \multirow{4}{*}{$\mathrm{T}_{1}$} & $\mathrm{P}_{1}$ & $1.49 \pm 0.02$ & $1.35 \pm 0.34$ & $1.28 \pm 0.06$ & $1.05 \pm 0.01$ & $0.81 \pm 0.03$ \\
\hline & & $\mathrm{P}_{2}$ & $1.49 \pm 0.02$ & $1.33 \pm 0.51$ & $1.26 \pm 0.04$ & $1.02 \pm 0.02$ & $0.82 \pm 0.12$ \\
\hline & & $\mathrm{P}_{3}$ & $1.49 \pm 0.02$ & $1.35 \pm 0.94$ & $1.26 \pm .02$ & $1.03 \pm 0.01$ & $0.82 \pm 0.15$ \\
\hline & & $\mathrm{P}_{4}$ & $1.49 \pm 0.02$ & $1.34 \pm 0.26$ & $1.20 \pm 0.03$ & $1.02 \pm 0.05$ & $0.83 \pm 0.02$ \\
\hline & \multirow{4}{*}{$\mathrm{T}_{2}$} & $\mathrm{P}_{1}$ & $1.68 \pm 0.11$ & $1.48 \pm 0.21$ & $1.33 \pm 0.03$ & $1.21 \pm 0.04$ & $0.97 \pm 0.03$ \\
\hline & & $\mathrm{P}_{2}$ & $1.68 \pm 0.11$ & $1.48 \pm 0.05$ & $1.33 \pm 0.02$ & $1.22 \pm 0.05$ & $0.98 \pm 0.01$ \\
\hline & & $\mathrm{P}_{3}$ & $1.68 \pm 0.11$ & $1.47 \pm 0.04$ & $1.33 \pm 0.01$ & $1.23 \pm 0.02$ & $0.97 \pm 0.02$ \\
\hline & & $\mathrm{P}_{4}$ & $1.68 \pm 0.11$ & $1.47 \pm 0.06$ & $\mathrm{I} .32 \pm 0.02$ & $1.23 \pm 0.03$ & $0.98 \pm 0.02$ \\
\hline & \multirow{4}{*}{$\mathrm{T}_{3}$} & $\mathrm{P}_{1}$ & $1.94 \pm 0.06$ & $1.81 \pm 0.01$ & $1.66 \pm 0.01$ & $1.37 \pm 0.02$ & $1.12 \pm 0.01$ \\
\hline & & $\mathrm{P}_{2}$ & $1.94 \pm 0.06$ & $1.82 \pm 0.06$ & $1.65 \pm 0.01$ & $1.35 \pm 0.01$ & $1.11 \pm 0.01$ \\
\hline & & $\mathrm{P}_{3}$ & $1.94 \pm 0.06$ & $1.82 \pm 0.04$ & $1.65 \pm 0.00$ & $1.36 \pm 0.03$ & $1.12 \pm 0.02$ \\
\hline & & $\mathrm{P}_{4}$ & $1.94 \pm 0.06$ & $1.83 \pm 0.02$ & $1.66 \pm 0.01$ & $1.36 \pm 0.05$ & $1.12 \pm 0.03$ \\
\hline & \multirow{4}{*}{$\mathrm{T}_{4}$} & $\mathrm{P}_{1}$ & $2.68 \pm 0.04$ & $2.24 \pm 0.09$ & $1.95 \pm 0.02$ & $1.81 \pm 0.05$ & $1.22 \pm 0.02$ \\
\hline & & $\mathrm{P}_{2}$ & $2.68 \pm 0.04$ & $2.25 \pm 0.08$ & $1.95 \pm 0.02$ & $1.82 \pm 0.02$ & $1.23 \pm 0.02$ \\
\hline & & $\mathrm{P}_{3}$ & $2.68 \pm 0.04$ & $2.25 \pm 0.05$ & $1.96 \pm 0.02$ & $1.82 \pm 0.02$ & $1.24 \pm 0.03$ \\
\hline & & $\mathrm{P}_{4}$ & $2.68 \pm 0.04$ & $2.24 \pm 0.11$ & $1.96 \pm 0.03$ & $1.83 \pm 0.04$ & $1.25 \pm 0.01$ \\
\hline
\end{tabular}

$\mathrm{CD}(\mathrm{p} \leq 0.05): \mathrm{V}: 0.08, \mathrm{~T}: 0.13, \mathrm{~S}: 1.15, \mathrm{P}: \mathrm{NS}, \mathrm{V} \times \mathrm{T}: 0.22, \mathrm{~V} \times \mathrm{S}: 0.19, \mathrm{~T} \times \mathrm{S}: 0.45, \mathrm{P} \times \mathrm{S}: \mathrm{NS}$

The ' $b$ ' values differ significantly in case of variety, treatment and storage period. Treatment increased the ' $b$ ' value to same level as that of ' $a$ ' value however the effect of storage in ' $b$ ' value was opposite to that of ' $a$ ' value. The ' $b$ ' value increased upon storage which concludes that yellowness increased during storage of brown rice. The b-values of parboiled brown rice ranged from 9.23-11.33. After soaking for $1-4 \mathrm{hr}$ at the initial temperatures of $70^{\circ} \mathrm{C}$ and $80^{\circ} \mathrm{C}$, followed by steaming time at 10-20 min, it was found that b-value increased for all three rice varieties. Moreover, b-value was affected by steaming time and soaking temperature. Parboiling was observed to decrease $L$ value and increase a and b-values (Mir and Bosco 2013). Among the varieties PR-118 had maximum b-values for $\mathrm{T}_{4}$ treatment among the other treatments as compared to Punjab Mehak and PR-115 of parboiled brown rice. The b-values for parboiled brown rice increased with increased storage periods.

At a given soaking time, higher soaking temperature gave higher b-value. Thus, increasing soaking temperature and time resulted in higher yellowness. Parboiling affects the color of milled rice. Upon altering different processing parameters including the soaking water temperature, soaking, and heating duration (Bhattacharya and Subba Rao 1966) color of parboiled brown rice 
changes from white to yellow. Normally, discoloration of parboiled rice was directly related with the market value because in most countries consumers do not accept dark colored parboiled rice. Changing in b-value of parboiled brown rice was mainly caused by Maillard type non-enzymatic browning (Kimura et al. 1993).
Pigments contribute the coloration of parboiled rice. Some nutrients from the bran leached out during parboiling (Ramalingham and Anthony 1996). Therefore, parboiled rice had poor qualities due to high heat treatments done during three main steps of parboiling process (soaking, steaming and drying) could be improved by parboiling at low heat treatments (Islam et al. 2002).

Table 6a Effect of variety, treatments, packaging material and storage period on amylose content (\%) of brown rice

\begin{tabular}{|c|c|c|c|c|c|c|c|}
\hline \multirow{2}{*}{ Variety } & \multirow{2}{*}{ Treatment } & \multirow{2}{*}{$\begin{array}{l}\text { Packaging } \\
\text { Material }\end{array}$} & \multicolumn{5}{|c|}{ Storage period } \\
\hline & & & $\mathbf{S}_{\mathbf{1}}$ & $\mathbf{S}_{2}$ & $\mathbf{S}_{3}$ & $\mathbf{S}_{4}$ & $\mathbf{S}_{5}$ \\
\hline \multirow{16}{*}{$\mathbf{V}_{1}$} & \multirow{4}{*}{$\mathrm{T}_{1}$} & $\mathrm{P}_{1}$ & $19.80 \pm 1.65$ & $19.54 \pm 1.22$ & $19.55 \pm 3.29$ & $17.76 \pm 1.05$ & $18.04 \pm 2.19$ \\
\hline & & $\mathrm{P}_{2}$ & $19.80 \pm 1.65$ & $19.53 \pm 1.36$ & $19.52 \pm 3.38$ & $17.79 \pm 1.09$ & $18.05 \pm 2.44$ \\
\hline & & $\mathrm{P}_{3}$ & $19.85 \pm 1.65$ & $19.52 \pm 1.49$ & $19.55 \pm 3.14$ & $17.74 \pm 1.11$ & $18.06 \pm 2.54$ \\
\hline & & $\mathrm{P}_{4}$ & $19.80 \pm 1.65$ & $19.56 \pm 1.51$ & $19.58 \pm 3.06$ & $17.73 \pm 1.24$ & $18.04 \pm 2.56$ \\
\hline & \multirow{4}{*}{$\mathrm{T}_{2}$} & $\mathrm{P}_{1}$ & $25.93 \pm 3.24$ & $23.25 \pm 2.36$ & $20.12 \pm 3.25$ & $20.00 \pm 2.26$ & $20.12 \pm 2.11$ \\
\hline & & $\mathrm{P}_{2}$ & $25.93 \pm 3.24$ & $23.28 \pm 1.50$ & $20.16 \pm 3.02$ & $20.15 \pm 2.51$ & $20.10 \pm 2.04$ \\
\hline & & $\mathrm{P}_{3}$ & $25.93 \pm 3.24$ & $23.24 \pm 2.59$ & $20.13 \pm 2.51$ & $20.18 \pm 2.45$ & $20.11 \pm 2.03$ \\
\hline & & $\mathrm{P}_{4}$ & $25.93 \pm 3.24$ & $23.25 \pm 2.51$ & $20.15 \pm 2.36$ & $20.12 \pm 2.59$ & $20.11 \pm 2.51$ \\
\hline & \multirow{4}{*}{$\mathrm{T}_{3}$} & $\mathrm{P}_{1}$ & $25.50 \pm 2.87$ & $24.47 \pm 3.26$ & $22.13 \pm 2.15$ & $20.74 \pm 2.51$ & $20.17 \pm 3.01$ \\
\hline & & $\mathrm{P}_{2}$ & $25.55 \pm 2.87$ & $24.85 \pm 3.55$ & $24.12 \pm 2.19$ & $23.78 \pm 2.16$ & $22.97 \pm 3.10$ \\
\hline & & $\mathrm{P}_{3}$ & $25.55 \pm 2.87$ & $24.00 \pm 3.48$ & $22.00 \pm 3.48$ & $20.00 \pm 2.48$ & $20.16 \pm 2.16$ \\
\hline & & $\mathrm{P}_{4}$ & $25.55 \pm 2.87$ & $25.00 \pm 3.61$ & $23.00 \pm 3.56$ & $21.00 \pm 2.17$ & $20.00 \pm 2.24$ \\
\hline & \multirow{4}{*}{$\mathrm{T}_{4}$} & $\mathrm{P}_{1}$ & $25.65 \pm 3.36$ & $24.85 \pm 3.24$ & $24.12 \pm 3.11$ & $23.78 \pm 3.16$ & $22.97 \pm 2.19$ \\
\hline & & $\mathrm{P}_{2}$ & $25.65 \pm 3.36$ & $24.13 \pm 3.58$ & $24.54 \pm 3.09$ & $23.72 \pm 3.25$ & $22.92 \pm 2.18$ \\
\hline & & $\mathrm{P}_{3}$ & $25.65 \pm 3.36$ & $24.82 \pm 3.78$ & $24.11 \pm 3.05$ & $24.75 \pm 3.54$ & $22.95 \pm 2.15$ \\
\hline & & $\mathrm{P}_{4}$ & $25.65 \pm 3.36$ & $24.81 \pm 2.54$ & $24.32 \pm 3.15$ & $23.65 \pm 3.21$ & $22.52 \pm 2.06$ \\
\hline \multirow{16}{*}{$\mathbf{V}_{2}$} & \multirow{4}{*}{$\mathrm{T}_{1}$} & $\mathrm{P}_{1}$ & $23.04 \pm 3.12$ & $22.90 \pm 2.15$ & $22.75 \pm 2.19$ & $22.09 \pm 3.11$ & $21.44 \pm 2.24$ \\
\hline & & $\mathrm{P}_{2}$ & $23.04 \pm 3.12$ & $22.00 \pm 2.35$ & $21.00 \pm 3.15$ & $21.43 \pm 3.26$ & $21.44 \pm 2.19$ \\
\hline & & $\mathrm{P}_{3}$ & $23.04 \pm 3.12$ & $23.00 \pm 2.64$ & $22.00 \pm 3.16$ & $21.00 \pm 2.56$ & $20.99 \pm 2.18$ \\
\hline & & $\mathrm{P}_{4}$ & $23.04 \pm 3.12$ & $22.54 \pm 2.48$ & $22.42 \pm 3.58$ & $22.00 \pm 2.51$ & $21.31 \pm 2.15$ \\
\hline & \multirow{4}{*}{$\mathrm{T}_{2}$} & $\mathrm{P}_{1}$ & $24.85 \pm 3.55$ & $22.65 \pm 1.26$ & $22.41 \pm 3.24$ & $22.40 \pm 2.22$ & $21.35 \pm 2.55$ \\
\hline & & $\mathrm{P}_{2}$ & $24.85 \pm 3.55$ & $24.47 \pm 2.54$ & $22.13 \pm 3.49$ & $20.74 \pm 2.36$ & $20.17 \pm 2.13$ \\
\hline & & $\mathrm{P}_{3}$ & $24.85 \pm 3.55$ & $22.90 \pm 2.58$ & $22.75 \pm 3.25$ & $20.00 \pm 2.16$ & $21.44 \pm 2.03$ \\
\hline & & $\mathrm{P}_{4}$ & $24.85 \pm 3.55$ & $23.00 \pm 5.51$ & $22.00 \pm 3.61$ & $21.54 \pm 2.64$ & $21.35 \pm 2.06$ \\
\hline & \multirow{4}{*}{$\mathrm{T}_{3}$} & $\mathrm{P}_{1}$ & $24.35 \pm 2.69$ & $24.05 \pm 2.65$ & $24.00 \pm 2.57$ & $23.75 \pm 2.18$ & $22.55 \pm 2.49$ \\
\hline & & $\mathrm{P}_{2}$ & $24.35 \pm 2.69$ & $24.00 \pm 3.15$ & $23.00 \pm 3.29$ & $22.95 \pm 2.51$ & $22.52 \pm 2.19$ \\
\hline & & $\mathrm{P}_{3}$ & $24.35 \pm 2.69$ & $24.00 \pm 3.25$ & $24.00 \pm 3.54$ & $23.00 \pm 2.49$ & $22.74 \pm 2.06$ \\
\hline & & $\mathrm{P}_{4}$ & $24.35 \pm 2.69$ & $24.13 \pm 3.64$ & $23.00 \pm 3.29$ & $23.47 \pm 3.12$ & $22.14 \pm 2.15$ \\
\hline & \multirow{4}{*}{$\mathrm{T}_{4}$} & $P_{1}$ & $25.12 \pm 4.15$ & $24.76 \pm 3.48$ & $24.12 \pm 3.15$ & $22.56 \pm 3.15$ & $22.04 \pm 3.16$ \\
\hline & & $\mathrm{P}_{2}$ & $25.12 \pm 4.15$ & $24.82 \pm 3.94$ & $24.11 \pm 2.58$ & $24.75 \pm 3.05$ & $22.95 \pm 3.12$ \\
\hline & & $\mathrm{P}_{3}$ & $25.12 \pm 4.15$ & $24.81 \pm 2.35$ & $24.32 \pm 2.61$ & $23.65 \pm 3.18$ & $22.52 \pm 2.44$ \\
\hline & & $\mathrm{P}_{4}$ & $25.12 \pm 4.15$ & $24.00 \pm 2.84$ & $23.00 \pm 2.38$ & $23.43 \pm 2.61$ & $22.13 \pm 2.15$ \\
\hline \multirow{16}{*}{$\mathbf{V}_{3}$} & \multirow{4}{*}{$\mathrm{T}_{1}$} & $\mathrm{P}_{1}$ & $22.08 \pm 3.08$ & $21.89 \pm 2.94$ & $21.77 \pm 2.59$ & $20.16 \pm 2.16$ & $20.99 \pm 2.51$ \\
\hline & & $\mathrm{P}_{2}$ & $22.08 \pm 3.08$ & $21.88 \pm 2.78$ & $21.75 \pm 2.57$ & $20.15 \pm 2.08$ & $20.82 \pm 2.57$ \\
\hline & & $\mathrm{P}_{3}$ & $22.08 \pm 3.08$ & $21.82 \pm 2.91$ & $21.76 \pm 2.29$ & $20.19 \pm 2.07$ & $20.81 \pm 2.45$ \\
\hline & & $\mathrm{P}_{4}$ & $22.08 \pm 3.08$ & $21.84 \pm 3.59$ & $21.72 \pm 3.91$ & $21.00 \pm 2.11$ & $20.76 \pm 2.36$ \\
\hline & \multirow{4}{*}{$\mathrm{T}_{2}$} & $P_{1}$ & $26.78 \pm 4.22$ & $25.48 \pm 3.15$ & $24.14 \pm 3.84$ & $23.79 \pm 2.05$ & $22.98 \pm 2.55$ \\
\hline & & $\mathrm{P}_{2}$ & $26.78 \pm 4.22$ & $25.56 \pm 3.29$ & $24.17 \pm 3.57$ & $23.74 \pm 2.16$ & $22.96 \pm 2.15$ \\
\hline & & $\mathrm{P}_{3}$ & $26.78 \pm 4.22$ & $25.63 \pm 3.61$ & $24.15 \pm 3.65$ & $23.71 \pm 2.48$ & $22.76 \pm 2.18$ \\
\hline & & $\mathrm{P}_{4}$ & $26.78 \pm 4.22$ & $25.71 \pm 3.54$ & $24.11 \pm 3.22$ & $23.71 \pm 3.02$ & $22.67 \pm 2.14$ \\
\hline & \multirow{4}{*}{$\mathrm{T}_{3}$} & $\mathrm{P}_{1}$ & $26.00 \pm 4.36$ & $25.78 \pm 3.58$ & $24.74 \pm 3.21$ & $23.89 \pm 3.16$ & $22.19 \pm 2.19$ \\
\hline & & $\mathrm{P}_{2}$ & $26.00 \pm 4.36$ & $25.71 \pm 4.19$ & $24.76 \pm 3.26$ & $23.88 \pm 3.08$ & $22.19 \pm 2.18$ \\
\hline & & $\mathrm{P}_{3}$ & $26.00 \pm 4.36$ & $25.72 \pm 3.15$ & $24.78 \pm 3.68$ & $23.85 \pm 3.16$ & $22.16 \pm 2.16$ \\
\hline & & $\mathrm{P}_{4}$ & $26.00 \pm 4.36$ & $25.74 \pm 3.49$ & $24.79 \pm 3.57$ & $23.8 \pm 2.05$ & $22.15 \pm 2.05$ \\
\hline & \multirow{4}{*}{$\mathrm{T}_{4}$} & $\mathrm{P}_{1}$ & $26.74 \pm 3.15$ & $26.09 \pm 4.10$ & $25.55 \pm 3.54$ & $24.71 \pm 2.04$ & $23.76 \pm 2.14$ \\
\hline & & $\mathrm{P}_{2}$ & $26.74 \pm 3.15$ & $26.00 \pm 4.09$ & $25.53 \pm 3.16$ & $24.00 \pm 2.16$ & $23.50 \pm 2.16$ \\
\hline & & $\mathrm{P}_{3}$ & $26.74 \pm 3.15$ & $26.00 \pm 3.25$ & $25.55 \pm 3.25$ & $24.00 \pm 2.01$ & $23.71 \pm 2.15$ \\
\hline & & $\mathrm{P}_{4}$ & $26.74 \pm 3.15$ & $26.06 \pm 3.62$ & $25.58 \pm 3.66$ & $24.50 \pm 2.06$ & $23.82 \pm 3.02$ \\
\hline
\end{tabular}

Effect of variety, treatments, packaging material and storage period on chemical composition of parboiled brown rice 
Varieties differed significantly in their protein contents. PR-115 had highest protein content, followed by Punjab Mehak and PR-118 respectively. Storage and packaging material had insignificant effect on the protein content of samples, though the data indicated a slight difference in protein content at different storage period. The individual effect of variety and treatment was found to be significant while storage period and packaging material had insignificant effect on protein content $(\%)$ of parboiled brown rice. The interaction of varieties with treatment, varieties with storage period and treatments with storage period had significant effect while interaction of packaging materials with storage period had insignificant effect on protein content $(\%)$ of parboiled brown rice. The protein content $(\%)$ remains constant during storage periods.

Table $6 \mathbf{b}$ Effect of variety, treatments, packaging material and storage period on free fatty acids (\% oleic acid) of brown rice

\begin{tabular}{|c|c|c|c|c|c|c|c|}
\hline \multirow[t]{2}{*}{ Variety } & \multirow[t]{2}{*}{ Treatment } & \multirow{2}{*}{$\begin{array}{l}\text { Packaging } \\
\text { Material }\end{array}$} & \multicolumn{3}{|c|}{ Storage Period } & \multirow[b]{2}{*}{$\mathbf{S}_{4}$} & \multirow[b]{2}{*}{$\mathbf{S}_{5}$} \\
\hline & & & $\mathbf{S}_{1}$ & $\mathbf{S}_{2}$ & $\mathbf{S}_{\mathbf{3}}$ & & \\
\hline \multirow{16}{*}{$\mathrm{V}_{1}$} & \multirow{4}{*}{$\mathrm{T}_{1}$} & $\mathrm{P}_{1}$ & $0.27 \pm 0.03$ & $0.28 \pm 0.02$ & $0.75 \pm 0.03$ & $0.79 \pm 0.04$ & $0.85 \pm 0.03$ \\
\hline & & $\mathrm{P}_{2}$ & $0.27 \pm 0.03$ & $0.29 \pm 0.03$ & $0.77 \pm 0.03$ & $0.81 \pm 0.04$ & $0.87 \pm 0.02$ \\
\hline & & $\mathrm{P}_{3}$ & $0.27 \pm 0.03$ & $0.27 \pm 0.02$ & $0.74 \pm 0.03$ & $0.75 \pm 0.04$ & $0.82 \pm 0.04$ \\
\hline & & $\mathrm{P}_{4}$ & $0.27 \pm 0.03$ & $0.28 \pm 0.02$ & $0.73 \pm 0.04$ & $0.76 \pm 0.05$ & $0.83 \pm 0.04$ \\
\hline & \multirow{4}{*}{$\mathrm{T}_{2}$} & $\mathrm{P}_{1}$ & $0.08 \pm 0.01$ & $0.09 \pm 0.01$ & $0.15 \pm 0.01$ & $0.17 \pm 0.01$ & $0.23 \pm 0.02$ \\
\hline & & $\mathrm{P}_{2}$ & $0.08 \pm 0.01$ & $0.12 \pm 0.00$ & $0.17 \pm 0.01$ & $0.19 \pm 0.01$ & $0.24 \pm 0.02$ \\
\hline & & $\mathrm{P}_{3}$ & $0.08 \pm 0.01$ & $0.08 \pm 0.00$ & $0.14 \pm 0.01$ & $0.18 \pm 0.02$ & $0.23 \pm 0.02$ \\
\hline & & $\mathrm{P}_{4}$ & $0.08 \pm 0.01$ & $0.10 \pm 0.01$ & $0.16 \pm 0.01$ & $0.17 \pm 0.02$ & $0.22 \pm 0.02$ \\
\hline & \multirow{4}{*}{$\mathrm{T}_{3}$} & $\mathrm{P}_{1}$ & $0.07 \pm 0.00$ & $0.08 \pm 0.01$ & $0.16 \pm 0.00$ & $0.19 \pm 0.02$ & $0.24 \pm 0.02$ \\
\hline & & $\mathrm{P}_{2}$ & $0.07 \pm 0.00$ & $0.09 \pm 0.00$ & $0.18 \pm 0.02$ & $0.21 \pm 0.01$ & $0.25 \pm 0.01$ \\
\hline & & $\mathrm{P}_{3}$ & $0.07 \pm 0.00$ & $0.08 \pm 0.02$ & $0.14 \pm 0.02$ & $0.18 \pm 0.01$ & $0.22 \pm 0.02$ \\
\hline & & $\mathrm{P}_{4}$ & $0.07 \pm 0.00$ & $0.09 \pm 0.01$ & $0.17 \pm 0.02$ & $0.19 \pm 0.01$ & $0.23 \pm 0.01$ \\
\hline & \multirow{4}{*}{$\mathrm{T}_{4}$} & $\mathrm{P}_{1}$ & $0.08 \pm 0.00$ & $0.10 \pm 0.01$ & $0.18 \pm 0.01$ & $0.20 \pm 0.02$ & $0.21 \pm 0.02$ \\
\hline & & $\mathrm{P}_{2}$ & $0.08 \pm 0.00$ & $0.13 \pm 0.00$ & $0.19 \pm 0.01$ & $0.20 \pm 0.01$ & $0.22 \pm 0.02$ \\
\hline & & $\mathrm{P}_{3}$ & $0.08 \pm 0.00$ & $0.11 \pm 0.00$ & $0.17 \pm 0.01$ & $0.18 \pm 0.01$ & $0.21 \pm 0.03$ \\
\hline & & $\mathrm{P}_{4}$ & $0.08 \pm 0.00$ & $0.11 \pm 0.01$ & $0.18 \pm 0.01$ & $0.20 \pm 0.01$ & $0.21 \pm 0.02$ \\
\hline \multirow{16}{*}{$\mathrm{V}_{2}$} & \multirow{4}{*}{$\mathrm{T}_{1}$} & $\mathrm{P}_{1}$ & $0.22 \pm 0.01$ & $0.23 \pm 0.02$ & $0.64 \pm 0.03$ & $0.71 \pm 0.03$ & $0.79 \pm 0.05$ \\
\hline & & $\mathrm{P}_{2}$ & $0.22 \pm 0.01$ & $0.27 \pm 0.03$ & $0.69 \pm 0.04$ & $0.76 \pm 0.03$ & $0.81 \pm 0.05$ \\
\hline & & $\mathrm{P}_{3}$ & $0.22 \pm 0.01$ & $0.25 \pm 0.03$ & $0.63 \pm 0.04$ & $0.70 \pm 0.02$ & $0.78 \pm 0.04$ \\
\hline & & $\mathrm{P}_{4}$ & $0.22 \pm 0.01$ & $0.24 \pm 0.02$ & $0.64 \pm 0.03$ & $0.71 \pm 0.05$ & $0.79 \pm 0.05$ \\
\hline & \multirow{4}{*}{$\mathrm{T}_{2}$} & $\mathrm{P}_{1}$ & $0.08 \pm 0.01$ & $0.09 \pm 0.01$ & $0.14 \pm 0.02$ & $0.19 \pm 0.00$ & $0.24 \pm 0.02$ \\
\hline & & $\mathrm{P}_{2}$ & $0.08 \pm 0.01$ & $0.11 \pm 0.01$ & $0.15 \pm 0.01$ & $0.21 \pm 0.01$ & $0.24 \pm 0.01$ \\
\hline & & $\mathrm{P}_{3}$ & $0.08 \pm 0.01$ & $0.10 \pm 0.01$ & $0.13 \pm 0.01$ & $0.19 \pm 0.01$ & $0.23 \pm 0.01$ \\
\hline & & $\mathrm{P}_{4}$ & $0.08 \pm 0.01$ & $0.11 \pm 0.01$ & $0.13 \pm 0.01$ & $0.18 \pm 0.01$ & $0.24 \pm 0.01$ \\
\hline & \multirow{4}{*}{$\mathrm{T}_{3}$} & $\mathrm{P}_{1}$ & $0.07 \pm 0.01$ & $0.08 \pm 0.01$ & $0.16 \pm 0.01$ & $0.19 \pm 0.01$ & $0.23 \pm 0.02$ \\
\hline & & $\mathrm{P}_{2}$ & $0.07 \pm 0.01$ & $0.09 \pm 0.01$ & $0.18 \pm 0.02$ & $0.21 \pm 0.01$ & $0.24 \pm 0.02$ \\
\hline & & $\mathrm{P}_{3}$ & $0.07 \pm 0.01$ & $0.08 \pm 0.01$ & $0.15 \pm 0.01$ & $0.18 \pm 0.01$ & $0.23 \pm 0.02$ \\
\hline & & $\mathrm{P}_{4}$ & $0.07 \pm 0.01$ & $0.08 \pm 0.01$ & $0.17 \pm 0.00$ & $0.19 \pm 0.01$ & $0.22 \pm 0.02$ \\
\hline & \multirow{4}{*}{$\mathrm{T}_{4}$} & $\mathrm{P}_{1}$ & $0.06 \pm 0.01$ & $0.10 \pm 0.01$ & $0.18 \pm 0.01$ & $0.20 \pm 0.02$ & $0.21 \pm 0.02$ \\
\hline & & $\mathrm{P}_{2}$ & $0.06 \pm 0.01$ & $0.12 \pm 0.01$ & $0.19 \pm 0.01$ & $0.21 \pm 0.02$ & $0.22 \pm 0.03$ \\
\hline & & $\mathrm{P}_{3}$ & $0.06 \pm 0.01$ & $0.09 \pm 0.00$ & $0.17 \pm 0.02$ & $0.19 \pm 0.02$ & $0.21 \pm 0.02$ \\
\hline & & $\mathrm{P}_{4}$ & $0.06 \pm 0.01$ & $0.11 \pm 0.01$ & $0.19 \pm 0.01$ & $0.20 \pm 0.02$ & $0.21 \pm 0.02$ \\
\hline \multirow{16}{*}{$\mathrm{V}_{3}$} & \multirow{4}{*}{$\mathrm{T}_{1}$} & $\mathrm{P}_{1}$ & $0.25 \pm 0.02$ & $0.27 \pm 0.02$ & $0.73 \pm 0.05$ & $0.77 \pm 0.03$ & $0.82 \pm 0.04$ \\
\hline & & $\mathrm{P}_{2}$ & $0.25 \pm 0.02$ & $0.29 \pm 0.02$ & $0.75 \pm 0.04$ & $0.79 \pm 0.03$ & $0.83 \pm .0 .06$ \\
\hline & & $\mathrm{P}_{3}$ & $0.25 \pm 0.02$ & $0.27 \pm 0.02$ & $0.74 \pm 0.04$ & $0.77 \pm 0.04$ & $0.81 \pm 0.04$ \\
\hline & & $\mathrm{P}_{4}$ & $0.25 \pm 0.02$ & $0.28 \pm 0.02$ & $0.74 \pm 0.03$ & $0.78 \pm 0.05$ & $0.82 \pm 0.05$ \\
\hline & \multirow{4}{*}{$\mathrm{T}_{2}$} & $\mathrm{P}_{1}$ & $0.03 \pm 0.01$ & $0.05 \pm 0.01$ & $0.11 \pm 0.01$ & $0.14 \pm 0.01$ & $0.19 \pm 0.01$ \\
\hline & & $\mathrm{P}_{2}$ & $0.03 \pm 0.01$ & $0.07 \pm 0.01$ & $0.13 \pm 0.01$ & $0.15 \pm 0.01$ & $0.20 \pm 0.02$ \\
\hline & & $\mathrm{P}_{3}$ & $0.03 \pm 0.01$ & $0.04 \pm 0.01$ & $0.12 \pm 0.02$ & $0.14 \pm 0.01$ & $0.18 \mathrm{~A} 0.01$ \\
\hline & & $\mathrm{P}_{4}$ & $0.03 \pm 0.01$ & $0.05 \pm 0.01$ & $0.13 \pm 0.02$ & $0.15 \pm 0.01$ & $0.19 \pm 0.01$ \\
\hline & \multirow{4}{*}{$\mathrm{T}_{3}$} & $\mathrm{P}_{1}$ & $0.03 \pm 0.01$ & $0.05 \pm 0.02$ & $0.11 \pm 0.01$ & $0.14 \pm 0.01$ & $0.19 \pm 0.01$ \\
\hline & & $\mathrm{P}_{2}$ & $0.03 \pm 0.01$ & $0.07 \pm 0.01$ & $0.12 \pm 0.01$ & $0.15 \pm 0.01$ & $0.20 \pm 0.01$ \\
\hline & & $\mathrm{P}_{3}$ & $0.03 \pm 0.01$ & $0.04 \pm 0.01$ & $0.10 \pm 0.00$ & $0.14 \pm 0.01$ & $0.19 \pm 0.02$ \\
\hline & & $\mathrm{P}_{4}$ & $0.03 \pm 0.01$ & $0.05 \pm 0.01$ & $0.12 \pm 0.01$ & $0.15 \pm 0.01$ & $0.19 \pm 0.01$ \\
\hline & & $\mathrm{P}_{1}$ & $0.02 \pm 0.01$ & $0.06 \pm 0.01$ & $0.12 \pm 0.01$ & $0.16 \pm 0.02$ & $0.18 \pm 0.02$ \\
\hline & $\mathrm{T}$ & $\mathrm{P}_{2}$ & $0.02 \pm 0.01$ & $0.07 \pm 0.01$ & $0.13 \pm 0.01$ & $0.17 \pm 0.02$ & $0.18 \pm 0.01$ \\
\hline & $\mathbf{T}_{4}$ & $\mathrm{P}_{3}$ & $0.02 \pm 0.01$ & $0.05 \pm 0.01$ & $0.10 \pm 0.02$ & $0.14 \pm 0.01$ & $0.18 \pm 0.01$ \\
\hline & & $\mathrm{P}_{4}$ & $0.02 \pm 0.01$ & $0.06 \pm 0.01$ & $0.11 \pm 0.01$ & $0.15 \pm 0.01$ & $0.18 \pm 0.01$ \\
\hline
\end{tabular}

$\mathrm{CD}(\mathrm{p} \leq 0.05): \mathrm{V}: 0.65, \mathrm{~T}: 0.75, \mathrm{~S}: 0.84, \mathrm{P}: \mathrm{NS}, \mathrm{V} \times \mathrm{T}: 0.13, \mathrm{~T} \times \mathrm{S}: 0.168, \mathrm{~V} \times \mathrm{S}: \mathrm{NS}, \mathrm{P} \times \mathrm{S}: \mathrm{NS}$

Mitra and Suryanarayan (1979) reported that protein content of parboiled brown rice was approximately 90 per cent of the level found in milled raw rice. The variable temperature treatment during parboiling of rice resulted in changes in protein and starch conformation was reported by Himmelsbach et al. (2005) 
There was decrease in protein content of the parboiled rice samples compared to the non- parboiled samples, which might be due to leaching of protein substances during soaking and rupturing that occurs in the molecules due to steaming. The process of parboiling makes the protein bodies to sink into the compact mass of gelatinized starch grains, making it less extractable hence a decrease in the protein content. There was no soaking or steaming process for the non-parboiled samples though little loss in protein content may occur during milling, but this was incomparable to what happens during parboiling, hence it had a higher protein content than the parboiled samples (Otegbayo et al. 2001).

Table 6c Effect of variety, treatments, packaging material and storage period on peroxide value (meq/kg) of brown rice

\begin{tabular}{|c|c|c|c|c|c|c|c|}
\hline \multirow{2}{*}{ Variety } & \multirow{2}{*}{ Treatment } & \multirow{2}{*}{$\begin{array}{l}\text { Packaging } \\
\text { Material }\end{array}$} & \multicolumn{5}{|c|}{ Storage Period } \\
\hline & & & $S_{1}$ & $\mathbf{S}_{2}$ & $\mathbf{S}_{3}$ & $\mathbf{S}_{4}$ & $\mathbf{S}_{5}$ \\
\hline \multirow{16}{*}{$\mathrm{V}_{1}$} & \multirow{4}{*}{$\mathrm{T}_{1}$} & $\mathrm{P}_{1}$ & $0.79 \pm 0.03$ & $0.80 \pm 0.03$ & $0.81 \pm 0.02$ & $0.88 \pm 0.03$ & $0.94 \pm 0.04$ \\
\hline & & $\mathrm{P}_{2}$ & $0.79 \pm 0.03$ & $0.81 \pm 0.03$ & $0.83 \pm 0.03$ & $0.89 \pm 0.03$ & $0.95 \pm 0.05$ \\
\hline & & $\mathrm{P}_{3}$ & $0.79 \pm 0.03$ & $0.79 \pm 0.02$ & $0.82 \pm 0.03$ & $0.87 \pm 0.04$ & $0.94 \pm 0.05$ \\
\hline & & $\mathrm{P}_{4}$ & $0.79 \pm 0.03$ & $0.80 \pm 0.03$ & $0.82 \pm 0.03$ & $0.86 \pm 0.05$ & $0.93 \pm 0.05$ \\
\hline & \multirow{4}{*}{$\mathrm{T}_{2}$} & $\mathrm{P}_{1}$ & $0.66 \pm 0.03$ & $0.67 \pm 0.02$ & $0.68 \pm 0.02$ & $0.70 \pm 0.03$ & $0.72 \pm 0.03$ \\
\hline & & $\mathrm{P}_{2}$ & $0.66 \pm 0.03$ & $0.68 \pm 0.02$ & $0.70 \pm 0.02$ & $0.72 \pm 0.02$ & $0.72 \pm 0.03$ \\
\hline & & $\mathrm{P}_{3}$ & $0.66 \pm 0.03$ & $0.66 \pm 0.02$ & $0.68 \pm 0.02$ & $0.70 \pm 0.02$ & $0.71 \pm 0.03$ \\
\hline & & $\mathrm{P}_{4}$ & $0.66 \pm 0.03$ & $0.66 \pm 0.02$ & $0.69 \pm 0.02$ & $0.71 \pm 0.02$ & $0.71 \pm 0.02$ \\
\hline & \multirow{4}{*}{$\mathrm{T}_{3}$} & $\mathrm{P}_{1}$ & $0.69 \pm 0.04$ & $0.70 \pm 0.02$ & $0.72 \pm 0.02$ & $0.73 \pm 0.02$ & $0.73 \pm 0.02$ \\
\hline & & $\mathrm{P}_{2}$ & $0.69 \pm 0.04$ & $0.71 \pm 0.01$ & $0.72 \pm 0.02$ & $0.73 \pm 0.03$ & $0.74 \pm 0.03$ \\
\hline & & $\mathrm{P}_{3}$ & $0.69 \pm 0.04$ & $0.69 \pm 0.01$ & $0.69 \pm 0.02$ & $0.72 \pm 0.02$ & $0.73 \pm 0.02$ \\
\hline & & $\mathrm{P}_{4}$ & $0.69 \pm 0.04$ & $0.69 \pm 0.01$ & $0.70 \pm 0.02$ & $0.72 \pm 0.01$ & $0.72 \pm 0.02$ \\
\hline & \multirow{4}{*}{$\mathrm{T}_{4}$} & $\mathrm{P}_{1}$ & $0.56 \pm 0.02$ & $0.58 \pm 0.1$ & $0.59 \pm 0.01$ & $0.62 \pm 0.02$ & $0.64 \pm 0.03$ \\
\hline & & $\mathrm{P}_{2}$ & $0.56 \pm 0.02$ & $0.59 \pm 0.01$ & $0.60 \pm 0.01$ & $0.63 \pm 0.02$ & $0.65 \pm 0.02$ \\
\hline & & $\mathrm{P}_{3}$ & $0.56 \pm 0.02$ & $0.56 \pm 0.01$ & $0.58 \pm 0.02$ & $0.61 \pm 0.01$ & $0.63 \pm 0.02$ \\
\hline & & $\mathrm{P}_{4}$ & $0.56 \pm 0.02$ & $0.57 \pm 0.1$ & $0.57 \pm 0.02$ & $0.60 \pm 0.02$ & $0.63 \pm 0.02$ \\
\hline \multirow{16}{*}{$\mathrm{V}_{2}$} & \multirow{4}{*}{$\mathrm{T}_{1}$} & $\mathrm{P}_{1}$ & $0.79 \pm 0.03$ & $0.80 \pm 0.02$ & $0.82 \pm 0.03$ & $0.89 \pm 0.03$ & $0.91 \pm 0.02$ \\
\hline & & $\mathrm{P}_{2}$ & $0.79 \pm 0.03$ & $0.81 \pm 0.03$ & $0.82 \pm 0.04$ & $0.88 \pm 0.05$ & $0.92 \pm 0.04$ \\
\hline & & $\mathrm{P}_{3}$ & $0.79 \pm 0.03$ & $0.79 \pm 0.02$ & $0.83 \pm 0.03$ & $0.88 \pm 0.04$ & $0.91 \pm 0.04$ \\
\hline & & $\mathrm{P}_{4}$ & $0.79 \pm 0.03$ & $0.81 \pm 0.02$ & $0.82 \pm 0.03$ & $0.89 \pm 0.03$ & $0.90 \pm 0.04$ \\
\hline & \multirow{4}{*}{$\mathrm{T}_{2}$} & $\mathrm{P}_{1}$ & $0.67 \pm 0.02$ & $0.68 \pm 0.02$ & $0.69 \pm 0.01$ & $0.70 \pm 0.02$ & $0.72 \pm 0.03$ \\
\hline & & $\mathrm{P}_{2}$ & $0.67 \pm 0.02$ & $0.69 \pm 0.03$ & $0.70 \pm 0.01$ & $0.71 \pm 0.02$ & $0.73 \pm 0.03$ \\
\hline & & $\mathrm{P}_{3}$ & $0.67 \pm 0.02$ & $0.67 \pm 0.2$ & $0.69 \pm 0.02$ & $0.71 \pm 0.02$ & $0.72 \pm 0.03$ \\
\hline & & $\mathrm{P}_{4}$ & $0.67 \pm 0.02$ & $0.67 \pm 0.02$ & $0.68 \pm 0.03$ & $0.70 \pm 0.01$ & $0.71 \pm 0.03$ \\
\hline & \multirow{4}{*}{$\mathrm{T}_{3}$} & $\mathrm{P}_{1}$ & $0.70 \pm 0.02$ & $0.70 \pm 0.02$ & $0.73 \pm 0.02$ & $0.74 \pm 0.02$ & $0.76 \pm 0.02$ \\
\hline & & $\mathrm{P}_{2}$ & $0.70 \pm 0.02$ & $0.73 \pm 0.02$ & $0.75 \pm 0.03$ & $0.76 \pm 0.03$ & $0.77 \pm 0.02$ \\
\hline & & $\mathrm{P}_{3}$ & $0.70 \pm 0.02$ & $0.70 \pm 0.03$ & $0.72 \pm 0.03$ & $0.73 \pm 0.02$ & $0.75 \pm 0.03$ \\
\hline & & $\mathrm{P}_{4}$ & $0.70 \pm 0.02$ & $0.70 \pm 0.03$ & $0.70 \pm 0.04$ & $0.74 \pm 0.02$ & $0.76 \pm 0.03$ \\
\hline & \multirow{4}{*}{$\mathrm{T}_{4}$} & $\mathrm{P}_{1}$ & $0.54 \pm 0.01$ & $0.57 \pm 0.01$ & $0.60 \pm 0.03$ & $0.62 \pm 0.03$ & $0.66 \pm 0.02$ \\
\hline & & $\mathrm{P}_{2}$ & $0.54 \pm 0.01$ & $0.57 \pm 0.01$ & $0.62 \pm 0.02$ & $0.62 \pm 0.02$ & $0.66 \pm 0.03$ \\
\hline & & $\mathrm{P}_{3}$ & $0.54 \pm 0.01$ & $0.58 \pm 0.01$ & $0.59 \pm 0.02$ & $0.63 \pm 0.01$ & $0.65 \pm 0.02$ \\
\hline & & $\mathrm{P}_{4}$ & $0.54 \pm 0.01$ & $0.58 \pm 0.02$ & $0.59 \pm 0.02$ & $0.61 \pm 0.01$ & $0.66 \pm 0.02$ \\
\hline \multirow{16}{*}{$\mathrm{V}_{3}$} & \multirow{4}{*}{$\mathrm{T}_{1}$} & $\mathrm{P}_{1}$ & $0.78 \pm 0.03$ & $0.80 \pm 0.03$ & $0.80 \pm 0.02$ & $0.86 \pm 0.03$ & $0.90 \pm 0.05$ \\
\hline & & $\mathrm{P}_{2}$ & $0.78 \pm 0.03$ & $0.82 \pm 0.03$ & $0.85 \pm 0.03$ & $0.88 \pm 0.02$ & $0.91 \pm 0.04$ \\
\hline & & $\mathrm{P}_{3}$ & $0.78 \pm 0.03$ & $0.78 \pm 0.03$ & $0.81 \pm 0.03$ & $0.85 \pm 0.02$ & $0.90 \pm 0.06$ \\
\hline & & $\mathrm{P}_{4}$ & $0.78 \pm 0.03$ & $0.78 \pm 0.03$ & $0.82 \pm 0.04$ & $0.86 \pm 0.01$ & $0.89 \pm 0.03$ \\
\hline & \multirow{4}{*}{$\mathrm{T}_{2}$} & $\mathrm{P}_{1}$ & $0.64 \pm 0.03$ & $0.64 \pm 0.02$ & $0.66 \pm 0.03$ & $0.69 \pm 0.01$ & $0.71 \pm 0.03$ \\
\hline & & $\mathrm{P}_{2}$ & $0.64 \pm 0.03$ & $0.65 \pm 0.02$ & $0.68 \pm 0.02$ & $0.71 \pm 0.02$ & $0.72 \pm 0.03$ \\
\hline & & $\mathrm{P}_{3}$ & $0.64 \pm 0.03$ & $0.64 \pm 0.02$ & $0.65 \pm 0.02$ & $0.65 \pm 0.02$ & $0.70 \pm 0.03$ \\
\hline & & $\mathrm{P}_{4}$ & $0.64 \pm 0.03$ & $0.64 \pm 0.03$ & $0.67 \pm 0.01$ & $0.66 \pm 0.02$ & $0.71 \pm 0.02$ \\
\hline & \multirow{4}{*}{$\mathrm{T}_{3}$} & $\mathrm{P}_{1}$ & $0.67 \pm 0.02$ & $0.69 \pm 0.02$ & $0.71 \pm 0.02$ & $0.72 \pm 0.02$ & $0.73 \pm 0.02$ \\
\hline & & $\mathrm{P}_{2}$ & $0.67 \pm 0.02$ & $0.68 \pm 0.01$ & $0.72 \pm 0.03$ & $0.73 \pm 0.03$ & $0.74 \pm 0.03$ \\
\hline & & $\mathrm{P}_{3}$ & $0.67 \pm 0.02$ & $0.67 \pm 0.02$ & $0.71 \pm 0.03$ & $0.71 \pm 0.03$ & $0.73 \pm 0.03$ \\
\hline & & $\mathrm{P}_{4}$ & $0.67 \pm 0.02$ & $0.66 \pm 0.02$ & $0.70 \pm 0.03$ & $0.72 \pm 0.02$ & $0.72 \pm 0.03$ \\
\hline & \multirow{4}{*}{$\mathrm{T}_{4}$} & $\mathrm{P}_{1}$ & $0.53 \pm 0.02$ & $0.56 \pm 0.01$ & $0.57 \pm 0.03$ & $0.60 \pm 0.02$ & $0.62 \pm 0.03$ \\
\hline & & $\mathrm{P}_{2}$ & $0.53 \pm 0.02$ & $0.57 \pm 0.01$ & $0.57 \pm 0.02$ & $0.62 \pm 0.02$ & $0.64 \pm 0.02$ \\
\hline & & $\mathrm{P}_{3}$ & $0.53 \pm 0.02$ & $0.53 \pm 0.02$ & $0.55 \pm 0.01$ & $0.61 \pm 0.02$ & $0.62 \pm 0.02$ \\
\hline & & $\mathrm{P}_{4}$ & $0.53 \pm 0.02$ & $0.53 \pm 0.02$ & $0.56 \pm 0.01$ & $0.59 \pm 0.01$ & $0.63 \pm 0.02$ \\
\hline
\end{tabular}

\section{Ash content (\%)}

No significant difference was found in ash content of variety due to treatment, storage and packaging material. Punjab Mehak had highest ash content followed by PR-115. Even none of the interaction was found to be significant. Values for ash content for treated brown rice were found to be within the range of 0.60 to 1.65 though no definite pattern could be observed in ash content. Ash content of all varieties was significantly different from each other and was observed in the range of 2.70 percent to 3.84 percent. The ash content $(\%)$ of parboiled brown rice remains constant during storage periods. Farhan (2011) reported that all the treatment had insignificant effect on ash content during storage periods.

\section{Crude fat}

The per cent crude fat content of hydrothermally treated brown rice showed significant differences among varieties and treatments. Among varieties PR-118 
had highest fat content followed by Punjab Mehak and PR-115 in the order. Fat content of brown rice decreased significantly due to hydrothermal treatments given to paddy, the decrease being more in $\mathrm{T}_{4}$ treatment among the other treatments. Storage period had insignificant impact on the fat content of brown rice. The interaction of treatment with variety was found to be significant while remaining all interactions were found to be insignificant.

The individual effect of variety and treatment was found to be significant while packaging material and storage period had insignificant effect on fat content (\%) of parboiled brown rice. Padua and Julaino (1974) reported that total fat in raw and treated brown rice remain unchanged over the storage period. The parboiled rice samples had lower fat content than the non-parboiled samples. Leaching and rupturing of oil globules during the parboiling process due to increase in temperature and steaming pressure (Otegbayo et al. 2001). Farhan (2011) found that bran fraction had a significantly high fat percentage for both raw and parboiled varieties in comparison with other fractions.

\section{Effect of variety, treatments, packaging material and storage period on amylose content (\%) of brown rice}

Amylose content was considered the single most important characteristics for predicting rice cooking and processing behavior. Amylose content of brown rice directly related to water absorption, volume expansion, fluffiness and separability of cooked grains. It inversely related to cohesiveness, tenderness and glossiness. The individual effect of variety, treatments and packaging material on amylose content (\%) of brown rice (Table 6.a) had significant variations while packaging materials had insignificant effect on amylose content (\%) of brown rice. Pujab Mehak had highest amylose content as compared to PR-115 and PR-118 varieties. Amylose content of brown rice increased with treatment and decreased with storage. $\mathrm{T}_{4}$ was found to be most effective treatment among the other treatments to increase the amylose content of brown rice over the control. Amylose content decreased during storage but the rate of decrease was very slow making varieties good enough in amylose content.

The interaction of varieties with treatment, varieties with storage period and treatments with storage period had significant effect while interaction of packaging materials with storage period had insignificant effect on amylose content (\%) of parboiled brown rice. The amylose content (\%) decreased with increased storage periods. Long grained variety Punjab Mehak had high amylose content and same findings were reported by Williams et al. (1958). Steaming and parboiling causes increased water soluble amylose fraction in parboiled rice than in raw rice as reported by Simpson et al. (1965) and Bhattacharya and Sowbhagya (1972). There was decrease in amylose content of the parboiled rice as compared to non- parboiled rice samples. This was because of starch solubilisation and leaching of the amylose molecules into the surrounding water during soaking and subsequent steaming during parboiling. The differences in chemical composition of the two rice varieties might be caused by variety and environmental factors such as location of field, planting season, time and rate of nitrogen fertilizer application, solar radiation during grain development, spacing and application of herbicides at sub-herbicida1 levels, location and structure of oil globules (Otegbayo et al. 2001).

Effect of variety, treatments, packaging material and storage period on free fatty acids (\% oleic acid) of brown rice

Free fatty acid content of grain was often taken as a measure of deterioration during storage. More the free fatty acid contents of grain during storage, higher the rate of deterioration. Significant variations were found in free fatty acid content among varieties (Table 6.b). Higher values of FFA were observed for varieties PR-115 followed by PR-118 and Punjab Mehak in the order. Hydrothermal treatments reduced the free fatty acid content significantly over the control. Treatment $T_{3}$ and $T_{4}$ proved to be equally effective in reducing the free fatty acid content of brown rice. Free fatty acid content increased significantly with storage period but in control more increase was recorded. Packaging showed insignificant differences in free fatty acid content; however the free fatty acid content seemed to be higher in $\mathrm{P}_{2}$ as compared to other packaging material showing that cloth bags deteriorated the grains rapidly. The individual effect of variety, treatment and storage period was found to be significant while packaging material had insignificant effect on free fatty acid content of parboiled brown rice. The interaction of varieties with treatment and varieties with storage period had significant effect while interaction of treatments with storage period and packaging materials with storage period had insignificant effect on free fatty acid content of parboiled brown rice. The free fatty acid content increased with increased storage periods.

Desikachar et al. (1969) reported that pressure parboiling reduced the free fatty acid to a greater extent for hydrothermally treated parboiled brown rice. Steaming process during parboiling might have been helping to reduce the free fatty acids. Itoh and Kawamura (1983) reported lower free fatty acid value for parboiled rice than the raw rice stored under similar conditions. The increase in free fatty acid during storage of brown rice has been reported in a number of studies. Guraya et al (2011) reported that average free fatty acid values were higher for long-grain brown rice and American Basmati brown rice; whereas, parboiled long-grain brown rice produced at least five times less free fatty acid during storage. Guraya (2011) proved that type of packaging materials had no effect on the formation of free fatty acid during storage. But all other treatments except packaging materials had a significant effect on the production of free fatty acid.

Effect of variety, treatments, packaging material and storage period on peroxide value (meq/kg) of brown rice

Peroxide value for hydrothermally treated brown rice was presented in Table 6.c. Values showed significant difference in peroxide content among varieties as influenced by treatments, storage period and packaging materials. Peroxide value was one of the indicators for deterioration of samples.

More the peroxide value more will be the deterioration rate. Samples stored in dark area exhibit less peroxide value as compared to samples exposed to light because environmental oxygen enhanced the rate of production of peroxide value. Results showed that treatment decreases the peroxide value of brown rice over control. Punjab Mehak had least peroxide value followed by PR-115 and PR-118 in the order. Among treatments $\mathrm{T}_{3}$ proved to be more effective in decreasing peroxide value as compared to other treatments of all varieties. Storage period had significant effect on peroxide value. The peroxide content increased upon storage; this increase was higher for control as compared to treated sample. Packaging material upto some extent had significant impact on peroxide value. Brown rice packed in transparent packaging showed higher increase in peroxide values due to exposure of samples to oxygen which gradually increases peroxide value. All interactions were found to be significant. The individual effect of variety, treatment, storage period and packaging material was found to be significant effect on peroxide content of parboiled brown rice. The interaction of varieties with treatment, varieties with storage period, treatment with storage period and packaging materials with storage period had significant effect on peroxide value of parboiled brown rice. The peroxide value increased with increased storage periods. An increase in peroxide value was observed during storage of brown rice .The peroxide value, free fatty acid contents, and carbonyl values have been adopted as indices for determining maximum storage periods (Suzuki et al. 1996). Marked increases in the head rice due to parboiling have been well recognized.

\section{CONCLUSIONS}

Significant differences were found in physico-chemical and color properties of treated brown rice compared to raw unparboiled brown rice. Brown rice percentage, head rice yield, bulk density, 1000 kernel weight and L/B ratio improved with hydrothermal treatments. Amylose content increased significantly with hydrothermal treatments improving quality of brown rice. Free fatty acid and peroxide content decreased significantly with treatments enhancing shelf life of brown rice. Hot water treatment followed by steaming for $15 \mathrm{~min}$ was found best treatment among all other treatments. Punjab Mehak gave best response to treatments and hence retained better functional properties upon storage. Packaging in plastic bag under vacuum was found to be the best packaging material for control however for treated samples experimental data showed that packaging material play no significant role. Overall treatments proved to be functional in improving quality and shelf life of brown rice.

\section{REFERENCES}

AACC (2000). Approved Methods of American Association of Cereal Chemists. $10^{\text {th }}$ ed. St. Paul, MN, USA. ISBN 978-1-891127-68-2. https://doi.org/10.1016/s0144$\underline{8617(01) 00358-7}$

Alizadeh, M. R., Minaei, S., Tavakoli, T. \& Khoshtaghaza, M. H. (2006). Effect of de-awning on physical properties of paddy. Pakistan Journal of Biology Science 9, 1726-1731. DOI: 10.3923/pjbs.2006.1726.1731

AOAC (2000) Official methods of analysis 16" ed. Association of Official Analytical Chemists Washington, USA.

Bhattacharya, K. R. \& Sombhagya, C. N. (1972). Water uptake by rice during Cooking. $\quad$ Cereal Science Today, 16, 420. https://scholar.google.com/scholar_lookup?title=Water\%20uptake\%20by\%20rice $\% 20$ during $\% 20$ cooking\&journal=Cereal $\% 20$ Science $\% 20$ Today \&volume $=16 \& p u$ blication_year $=1971 \&$ pages $=420$ -

424\&author=Bhattacharya\&author=Sowbhagya .

Bhattacharya, K. R., \& Subba Rao, P.V. (1966). Effect of processing conditions on quality of parboiled rice. Journal of Agriculture and Food Chemistry, 14, 476479. DOI: $10.1021 / \mathrm{jf} 60147 \mathrm{a} 008$.

Desikachar, H. S. R., Mahadevappa, M., \& Bhashyam, M. K. (1969). Influence of harvesting date and traditional threshing practices on grain yield and milling quality of paddy. Journal of Food Science Technology, 6, 263-266.

Farhan, S., Pasha, I., Anjum, F.M., Suleria, H. A. R. \& Farooq, M. (2011). Effect of parboiling on physico- chemical and cooking attributes of different rice cultivars. 
Internet Journal of Food Safety 13, 237-245. http://www.internetjfs.org/articles/EFFECT\%20OF\%20PARBOILING\%20ON\% 20PHYSICO-

CHEMICAL\%20\&\%20COOKING\%20ATTRIBUTES\%20OF\%20DIFFERENT \%20RICE\%20CULTIVARS.pdf

Guraya, H. S. \& Patindol, J.A. (2011). Storage stability of flour-blasted brown rice. Cereal Chemistry, 88, 56-63. DOI: 10.1094/CCHEM-10-09-0132.

Himmelsbach, D., Manful, J.T. \& Coker, R.D. (2005). Changes in Rice with Variable Temperature Parboiling: Thermal and Spectroscopic Assessment. Cereal Chemistry 85(3), 384-390. DOI: 10.1094/CCHEM-85-3-0384.

Islam, M.D., Shimizu, N., \& Kimura, T. (2002). Effect of processing conditions on thermal properties of parboiled rice. Food Science and Technology Research 8, 131-136. DOI: 10.3136/fstr.8.131

Itoh, K. \& Kawamura, S. (1983). Studies on parboiled rice 1. Processing conditions of parboiled rice and its quality. Journal of Japan Society Food Science Technology, 32, 471.

Juliano, B. O. (1971). A simplified assay for milled rice amylose. Cereal Science Today, 16, 334 https://www.researchgate.net/publication/275886661 A Simplified Assay for Milled-Rice Amylose

Kibuuka, G. K. S. (1979). Increase in yield and nutritive value of rice: Effect of treatment with solvent, irradiation and steam on the quality of whole rice. Informative Annual, Faeuldade de Engenharia de Alimentos e Agricola, Universidad Estadual deCampinas No.7, 23-27 (FSTA (1981) No. 1M8).

Kimura, T., Bhattacharya, K. R. \& Ali, S. Z. (1993). Discoloration characteristics of rice during parboiling (I): Effect of processing conditions on the color intensity of parboiled rice. Journal of Society of Agriculture Structures, 24, 23-30. https://www.jstage.jst.go.jp/article/sasj1971/24/3/24_3_153/_pdf

Mir, S. \& Bosco, S. (2013). Effect of soaking temperature on physical and functional properties of parboiled rice cultivars grown in temperate region of India. Food and Nutrition Science, 4, 282-288. DOI: 10.4236/fns.2013.43038.

Mitra, G. N. \& Suryanarayana, A. (1979). Effects of different methods of processing on the protein content and composition of some rice varieties lprotein content. In proceedings of the forst Indian Convention of Food Scientists and Technologists pp.44 No.45. (FSTA (1979) 12M 1389)

Otegbayo, B. O., Osamuel, F.,\& Fashakin, J. B. (2001). Effect of parboiling on physicochemical qualities of two local rice varieties in Nigeria. Journal of Food Technology Africa, 6, 130-132. DOI: 10.4314/jfta.v6i4.19305

Padua, A. R. \& Juliano, B. O. (1974). Effect of parboiling on thiamine, protein and fat content of rice. Journal of the Science Food Agriculture, 25, 697-700 (FSTA (1974) No.9M1264. DOI: 10.1002/jsfa.2740250611.

Parnsakhorn, S. \& Noomhorm, S. (2008). Changes in physicochemical properties of parboiled brown rice during heat treatment. Agricultural Engineering International: the CIGR E-journal. Manuscript FP 08 009. Vol. X. August. file:///C:/Users/admin/Downloads/Changes in Physicochemical Properties of P arboiled.pdf

Ramlingham, N. \& Anthony, R.S. (1996). Studies on the soak water characteristics in various in paddy parboiling methods. Bioresource Technology, 55, 259-261. https://doi.org/10.1016/0960-8524(96)00001-6.

Saif, S. M. H., Suter, D. A. \& Lan, Y. (2004). Effects of processing conditions and environmental exposure on the tensile properties of parboiled rice. Biosystems Engineering, 89, 321-330. DOI:10.1016/j.biosystemseng.2004.08.010

Sareepuang, K., Siri, S., Amornpun, Wiset, L. \& Meeso, N. (2008). Effect of soaking temperature on physical, chemical and cooking properties of parboiled fragrant rice. World Journal of Agricultural Science, 4, 409-415. https://pdfs.semanticscholar.org/7988/25cd372dbedf18033ac0d4224b33838386a0 .pdf

Shaheen, A. B., Dash, A. A. \& Shirbeeny, A. E. (1975). Effect of parboiling of rice on the rate of lipid hydrolysis and deterioration of rice bran. Cereal $\begin{array}{llllll}\text { Chemistry } & 52, & 1-80 & \text { (FSTA } & \text { (1975) } & \text { No.7M864). }\end{array}$ https://www.aaccnet.org/publications/cc/backissues/1975/Documents/chem52 1.p df

Simpson, J. E. (1965). Quality evaluation studies of foreign and domestic rice. Tech, Bull:1381.U.S D A A R S Washington, D C U S A.

Suzuki, Y.,Yasui, T., Matsukura, U. \& Terao, J. (1996). Oxidative stability of bran lipids from rice variety (Oryza sativa (L.)) lacking lipoxygenase- 3 in seeds. Journal of Agriculture and Food Chemistry, 44, 3479-83. DOI:10.1021/jf9600465

Tian, S., Nakamura, K. \& Kayahara, H. (2004). Analysis of phenolic compounds in white rice, brown rice and germinated brown rice. Agriculture and Food Chemistry, 52, 4808-4813. DOI: 10.1021/jf049446f

Vitti, P., Leitao, R. F. F. \& Dizzinatto, A. (1975). Parboiling rice varieties. Colentanea do Instituto de Technologia de Alimentes 6, 103-119 (FSTA (1976) No.5M618).

Williams, V. R., Wu, W. T., Tsai, H. Y. \& Bates, H. G. (1958). Varietal differences in amylose content of rice starch. Journal of Agriculture and Food Chemistry, 6, 47. DOI: 10.1021/jf60083a009 